\title{
Multiple Rotations of Gaussian Quadratures: An Efficient Method for Uncertainty Analyses in Large-Scale Simulation Models
}

Davit Stepanyan, ${ }^{1,2}$ Harald Grethe, ${ }^{2}$ Georg Zimmermann,,${ }^{3,7}$ Khalid Siddig, ${ }^{2}$ Andre

Deppermann, ${ }^{4}$ Arndt Feuerbacher, ${ }^{5}$ Jonas Luckmann, ${ }^{2}$ Hugo Valin, ${ }^{4}$ Takamasa Nishizawa, ${ }^{6}$

Tatiana Ermolieva, ${ }^{4}$ Petr Havlik ${ }^{4}$

$1 \quad$ Corresponding author: email: davit.stepanyan@hu-berlin.de; Tel.: +49 531 596-5147;

Fax: +49531596-5199

2 International Agricultural Trade and Development Group, Humboldt University of Berlin, Unter den Linden 6, 10099 Berlin, Germany

3 Institute of Applied Mathematics and Statistics, University of Hohenheim, Schloss Hohenheim, Westhof-Süd, 70599 Stuttgart, Germany

$4 \quad$ Ecosystems Services and Management Program, International Institute for Applied Systems Analysis, Schlossplatz 1, 2361 Laxenburg, Austria

5 Institute of Agricultural and Food Policy, University of Hohenheim, Schwerzstraße 46, 70593 Stuttgart, Germany

$6 \quad$ Farm Economics and Ecosystem Services Group, Leibniz Centre for Agricultural Landscape Research, Eberswalder Str. 84, 15374 Müncheberg, Germany

7 Computational Science Lab, University of Hohenheim, Steckfeldstraße 2, 70599 Stuttgart, Germany 


\begin{abstract}
Concerns regarding the impact of climate change, food price volatility, and weather uncertainty have motivated users of simulation models to consider uncertainty in their simulations. One way to do this is to integrate uncertainty components in the model equations, thus turning the model into a problem of numerical integration. Most of these problems do not have analytical solutions, and researchers, therefore, apply numerical approximation methods. This article presents a novel approach to conducting an uncertainty analysis as an alternative to the computationally burdensome Monte Carlo-based (MC) methods. The developed method is based on the degree three Gaussian quadrature (GQ) formulae and is tested using three large-scale simulation models. While a standard single GQ method often produces low-quality approximations, the results of this study demonstrate that the proposed approach reduces the approximation errors by a factor of nine using only $3.4 \%$ of the computational effort required by the MC-based methods in the most computationally demanding model.
\end{abstract}

Keywords: Uncertainty analysis, systematic sensitivity analysis, stochastic modeling, multiple rotations of Gaussian quadratures, Monte Carlo sampling, computable general equilibrium models, partial equilibrium models.

Software availability

Model name: MRGQ generator

Contact person: Davit Stepanyan, T. +49 531596 5147, E-mail address: davit.stepanyan@huberlin.de

Address: Unter den Linden 6, 10099 Berlin, Germany

Hardware: PC

Software: Wolfram Mathematica

Availability: Available free of charge: https://github.com/Davstepany/MRGQ.git

Model name: STAGE2 (static CGE)

Contact Person: Scott McDonald, T. +44 114250 9904, E-mail address: scott@cgemod.org.uk Address: 14 Moncrieffe Road, Sheffield, S7 1HR, UK

DATA: A 2012 SAM for Bhutan (Feuerbacher et al. 2017) 
Hardware: PC

Software: GAMS

Availability: The code is available upon request.

Model name: GLOBIOM

Contact Person: Petr Havlik, T. +43(0) 2236807 511, E-mail address: havlikpt@iiasa.ac.at Address: IIASA, Schlossplatz 1, A-2361 Laxenburg, Austria

DATA: FAOSTAT

Hardware: High-performance computational server available at IIASA

Software: GAMS

Availability: The code is available upon request.

Model name: Recursive Dynamic CGE

Contact Person: James Thurlow, T. +1 202-862-5600

Address: 2033 K Street NW, Washington DC 20006, United States

DATA: A post-separation SAM for the Sudan (Siddig et al. 2016). We deposited the input data of this model (Social Accounting Matrix for Sudan) in an online repository, which can be accessed through the following link: https://zenodo.org/record/3970060\#.XybWqCgzaUk Hardware: PC

Software: GAMS

Availability: The code is available upon request. 


\section{Introduction}

\subsection{Uncertainty in simulation modeling}

Simulation models are an established tool for assessing the impact of an exogenous shock, such as political or biophysical changes in ecological, economic, and social systems. Such models are also widely applied in analyses of agro-environmental systems and land-use changes. However, because all models are imperfect representations of real-world systems and accurate input data are not always available, the robustness of the model results needs to be addressed. In the context of uncertainty analysis (UA), Sheikholeslami et al. (2019) defined robustness as the stability of the results, i.e., a lower variability of the results obtained by solving a model with a sample of parameter values drawn from a probability distribution indicates a higher degree of robustness. Interested readers are directed to Kwakkel et al. (2016) for a comparison of different robustness metrics.

A standard approach to tackling uncertainty in simulation models depicting agro-environmental systems is to incorporate uncertain terms ${ }^{1}$ sampled from a probability distribution. This allows us to address not only issues of robustness but also a wide range of policy questions related to uncertainty. Simulation model analyses that use such terms to depict uncertainty can be classified into two main groups according to their purpose. The first group applies a systematic sensitivity analysis (SSA) regarding uncertain model parameters, typically referred to as epistemic uncertainty resulting from a lack of knowledge (Uusitalo et al. 2015) (e.g., Arndt and Hertel 1997; Valenzuela et al. 2007; Beckman et al. 2011; Villoria et al. 2013; Cho et al. 2016). The second group explicitly considers the uncertainties inherent in the input variables, such as the weather, by describing such variables with probability distributions and producing distributions of the model outputs under an input uncertainty (e.g., European Commission 2018; Lammoglia et al. 2018; OECD/FAO 2018). The latter group is a type of UA (Loucks and van Beek 2017). The uncertainty considered by the second group is known as aleatory uncertainty (Uusitalo et al. 2015). In simulating agro-environmental systems, many studies have addressed the policy implications of uncertainty (Westhoff et al. 2005; Hertel et al. 2010; Moss et al. 2010; Verma et al. 2011; Gouel

\footnotetext{
${ }^{1}$ Some studies refer to such uncertain terms as stochastic; see Beckman et al. (2011), Gouel and Jean (2013), and Pianosi et al. (2016).
} 
and Jean 2013). In this article, the term "uncertainty" refers to aleatory uncertainty because we consider the uncertainty resulting from crop yield variability to be due to weather uncertainty.

\subsection{Uncertainty analysis as a numerical integration problem}

To quantify uncertainty in simulation models, researchers normally apply numerical approximation methods because, in most cases, such problems do not have analytical solutions (Arndt 1996). One approach to modeling uncertainty is to consider it as a problem of numerical integration. Consider the following simple example of UA in a simulation model: Let $x$ be an exogenous variable or parameter, $g(x)$ be the probability density function describing the uncertainty of $x$ supported on a particular interval $[\mathrm{a}, \mathrm{b}]$, and $f(x)$ be a function in the model for which we wish to find the expected value:

(1) $E[f(x)]=\int_{a}^{b} f(x) g(x) d x$.

In many applications, such integrals cannot be evaluated directly because they are not given in a closed form. Instead, numerical integration methods must be used. To this end, we choose $n$ points $x_{k}$ (called nodes) within the domain of integration, with associated weights, $w_{k}$, and we approximate integral (1) using the following finite sum:

(2) $\tilde{E}[f(x)]=\sum_{k=1}^{n} f\left(x_{k}\right) w_{k}$.

The nodes and their weights for such a quadrature formula are chosen in such a way that approximation (2) yields the same results as (1) for polynomials of low degree. Consequently, the degree of accuracy of quadrature formula (2) is defined as follows:

(3) $\max \left\{M \in N_{0}: E\left[x^{m}\right]=\tilde{E}\left[x^{m}\right]\right.$ for $\left.m=0, \ldots, M\right\}$.

This approach approximates the continuous probability distribution with density function $g(x)$ in (1) based on a finite discrete probability distribution. Thus, the finite discrete probability 
distribution is chosen to maximize the number of shared moments (e.g., expected value, variance, skewness, or kurtosis) with a continuous probability distribution.

This approach can also be used for multivariate integrals; in this case, we refer to approximations of type (2) as cubature formulae. As an example, consider the case of a multivariate normal distribution with mean vector $\vec{\mu}$ and covariance matrix $\Sigma$. Then, $g(\vec{x})$ is given by the following:

(4) $g(\vec{x})=\frac{1}{\sqrt{(2 \pi)^{d} \operatorname{det}(\Sigma)}} \exp \left(-\frac{1}{2}(\vec{x}-\vec{\mu})^{T} \Sigma^{-1}(\vec{x}-\vec{\mu})\right)$.

Note, however, that this implies that the domain of integration is no longer bounded, but instead is all $R^{n}$ (Euclidean space). There is a wide range of methods for choosing the nodes and their weights. Those most frequently used are discussed below.

\subsection{Sampling and analysis methods}

Methods for SSA/UA can be categorized as local or global. Local methods consider the uncertainty of the model output against variations of a single input factor (Pianosi et al. 2016). The disadvantage of this approach is that it does not consider interactions among input factors and therefore only provides a limited view of model uncertainty (Douglas-Smith et al. 2020). By contrast, global methods evaluate the input uncertainty over the entire range of input space, varying all input factors simultaneously (Matott et al. 2009). This type of method allows for a more comprehensive depiction of model uncertainty by accounting for the interactions among the input factors (Saltelli and Annoni 2010). Saltelli et al. (2019) claim that SSA/UA should always be based on global methods because local methods do not adequately represent models with nonlinearities. However, considering the computational capacity required to produce statistically robust results, conventional methods of global SSA/UA suffer from poor computational efficiency (Razavi and Gupta 2016a), which is one of the factors explaining the limited use of these methods compared to local methods (Douglas-Smith et al. 2020).

Douglas-Smith et al. (2020) analyzed 11,625 studies applying or introducing SSA/UA techniques within the field of environmental science from 2000 to 2017. The study shows that, during the time period investigated, there was a sharp increase (by a factor of 5) in the number of publications 
that apply such techniques, whereas the trend of studies introducing and applying novel tools for SSA/UA has remained largely flat. Despite the fact that SSA/UA is becoming more relevant, researchers still apply conventional methods, which are computationally burdensome.

The remainder of this section discusses some of the most established and widely applied SSA/UA methods, presenting their advantages and drawbacks.

The Monte Carlo (MC) method was introduced by Metropolis and Ulam (1949) and has been one of the most commonly used sampling techniques. The basic idea behind the MC method is to perceive integration as a probabilistic problem and approximate its solution using statistical experiments. Thus, the underlying logic is to choose the nodes randomly. According to the law of large numbers, the numerical result will then be close to the correct value if the number of points is sufficiently large. Although this method is easy to apply and extremely effective, it is inefficient because it requires large sample sizes. According to Haber (1970), the MC sample size should range from 40,000 to 100,000, to obtain an error below $1 \%$. The main disadvantages of this method are therefore slow convergence rates with increasing sample sizes (Engels 1980) and high computational requirements. Because many iterations are necessary for obtaining reliable results (Artavia et al. 2015; Razavi and Gupta 2016a), its application in large-scale simulation models is extremely demanding, if not infeasible, in terms of the computational requirements, time, and data management costs (e.g., European Commission (2018)).

To achieve a higher convergence rate using MC-based methods, a type of stratified sampling is typically applied (Saltelli et al. 2008). The idea behind this approach is to divide the parameter space into sub-regions (strata) and assign an equal quota of samples to each sub-region (Norton 2015). In this case, the sub-regions do not necessarily need to be equally weighted. Hence, the sample size will be equal to $N \cdot S$, where $N$ is the number of strata, and $S$ is the number of points selected from each stratum. This strategy has several advantages over pure random sampling. First, it ensures that the randomly selected points are spread somewhat evenly across the domain of the distribution according to the probability mass, thus considerably increasing the rate of convergence. Consequently, the sample size required to obtain results of equal quality is much smaller than that used in random sampling. The challenge with this method is the definition of the strata and the calculation of their probabilities (Saltelli et al. 2008).

The Latin hypercube sampling (LHS) technique is a compromise between using pure random sampling and stratified sampling. It divides the domain of the probability distribution into $N$ 
subsets of equal probability, where $N$ is the sample size, and then randomly selects one point from each subset (Helton and Davis 2003). In contrast to stratified sampling, there is no need to define the strata or calculate their probabilities. This method ensures full coverage of the entire parameter space (Norton 2015).

As the main advantage of all MC-based methods, the accuracy of the approximation is independent of the degree of smoothness of the integration function. In addition, such methods are effective in revealing nonlinearities. Nonetheless, they require thousands of iterations for each input factor and can be extremely demanding computationally, particularly for large-scale simulation models (Razavi and Gupta 2016a).

Because no predetermined sample size fits all models, often - and typically driven by the computational feasibility — SSA/UA are applied in large-scale simulation models using ad hoc and relatively small sample sizes that may limit the quality of the approximations (Valin et al. 2015; OECD/FAO 2017; Villoria and Preckel 2017; Mary et al. 2018) ${ }^{2}$. This was also recognized by Sarrazin et al. (2016), who stated that in environmental applications with frequently complex models and computationally demanding simulations, a tradeoff exists between the robustness of the results and the computational costs ${ }^{3}$. Nevertheless, other studies have suggested various approaches to convergence evaluations. For example, Pianosi et al. (2016) suggest evaluating the convergence using sub-samples from the original sample and comparing the sensitivity indices of the results obtained from the sub-samples with the results obtained from the original sample. Yang (2011) suggests gradually increasing the sample size and observing the behavior of the coefficient of variation in the results.

Variance-based methods are designed to evaluate the impact of the variability of the input parameters on the overall variability of the output (Norton 2015). Some of the more well-known methods falling under this category are the Fourier amplitude sensitivity test (FAST) and the Sobol method (Douglas-Smith et al. 2020). Because these methods are based on the MC algorithm, they become computationally demanding as the number of input factors considered increases (Pianosi et al. 2016). For example, the Sobol method requires $N \cdot(2 n+2)$ points, where $N$ is the chosen MC sample, and $n$ is the number of input factors (Yang 2011). As an advantage of these methods,

\footnotetext{
2 These studies applied 550, 190, 300, and 10,000 points, respectively.

${ }^{3}$ However, it should be acknowledged that there are also studies that apply formal convergence evaluation criteria when conducting an uncertainty/sensitivity analysis; see Saltelli et al. (2010), Pianosi et al. (2016), and Razavi and Gupta (2016b).
} 
however, the properties of the model they are applied to do not influence the quality of the results (Saltelli et al. 2008).

Derivative-based methods can be viewed as extensions of local UA methods. The basic idea behind these methods is to compute the partial derivatives of the model output concerning each model input. Thus, these values can be interpreted as local sensitivity indices to rank the input factors according to their influence on the model output (Razavi et al. 2019). As the main disadvantage of these methods, the derivatives are only computed at the base points of the model inputs and do not provide information regarding the rest of the input space (Saltelli et al. 2008). The Morris method, also known as the elementary effects (EE) method, is a derivative-based approach (Morris 1991) that computes the partial derivatives of the model outputs with respect to a sample of randomly selected model inputs. Each sample's mean and standard deviation is then considered as an uncertainty measure. For example, a higher mean value indicates that the factor is important for the output, and a higher standard deviation indicates the nonlinearity of the factor for the output and a strong interaction with other factors (Norton 2015). The EE method requires $N \cdot(n+1)$ model evaluations, where $N$ is the MC sample size, and $n$ is the number of factors (Saltelli et al. 2008). This method has two main drawbacks. First, it is impossible to quantify the contribution of each factor to the output variability. Second, it is impossible to distinguish the factor nonlinearity from interactions with other factors (Yang 2011).

Two strategies can be applied when a UA is too computationally demanding. First, an emulator can be used as a low-degree substitute, and second, the efficiency of the computationally demanding method can be improved (Song et al. 2012).

According to O'Hagan (2006), an emulator is a statistical approximation of the original simulation model. If this approximation is sufficiently precise, it can substitute for the original simulation model in applying a costly SSA/UA. For example, Zhan et al. (2013) proposed a global SA analysis method that combines the Morris method with a statistical emulator to reduce the computational costs. Although other methods also exist, emulators are mostly based on Gaussian processes and represent a probability distribution for a desired function (O'Hagan 2012; Uusitalo et al. 2015). An emulator is based on a large sample of results from previously conducted simulations (O'Hagan 2012). According to Stanfill et al. (2015), emulator-based methods can accurately estimate the first-order sensitivity indices using half the number of computer model evaluations compared to 
traditional MC-based methods. A disadvantage of using an emulator is that it introduces numerical challenges related to model calibration and validation (Pianosi et al. 2016).

Gaussian quadratures (GQs) are a family of methods designed for efficiently and accurately approximating definite integrals (Arndt et al. 2015). Being a static estimator, GQ requires a minimal number of iterations ( $2 n$, where $n$ is the number of uncertain input factors) to reproduce the second central moments of a joint probability distribution (Haber 1970). According to Schürer (2003), the methods based on GQ are expected to be the most efficient for low-degree polynomials, i.e., for smooth integrands. This method is discussed more thoroughly in Section 2.

Other sampling approaches have also been designed for specific SSA/UA methods and are based on a simple random sampling, for example, sampling approaches for the FAST method (Cukier et al. 1973) and for calculating the Sobol sensitivity indices (Saltelli 2002). Interested readers are directed to a review by Gan et al. (2014).

As an important conclusion from this discussion on SSA/UA methods, there is always a compromise between the computational requirements and the output reliability when choosing the method. The choice of method does not depend solely on the dimensionality of the problem, but also on the smoothness of the integrand, i.e., the number of times the function is continuously differentiable (Arndt and Preckel 2006). GQ methods outperform MC-based methods in terms of efficiency and accuracy for smooth integrands. For highly nonlinear integrands, by contrast, MCbased methods may be more suitable because they are neither dependent on the smoothness of the integrand nor on the dimensionality of the problem (Schürer 2001, 2003).

After the initial uptake when applying the GQ methods in large-scale simulation models (Arndt and Hertel 1997; Valenzuela et al. 2007; Villoria et al. 2013), Artavia et al. (2015) found that the quality of the approximation differs depending on the initial position of the octahedron from which the rotation starts. In addition, a recent study by Villoria and Preckel (2017) pointed out inaccuracies in results based on GQ methods applied in the global trade analysis project (GTAP) model. Specifically, large differences have been found in the first three moments of the probability distributions of the results produced by GQ and MC. To address these inaccuracies, this article presents a novel approach to the reduction of the approximation error for GQ methods, called multiple rotations of Gaussian quadratures (MRGQ). The MRGQ method aims at improving the quality of the approximations using traditional GQ methods while keeping the computational 
requirements low. It is primarily designed for computationally demanding models, where the cost of applying MC-based methods without consideration of the emulators is prohibitively high ${ }^{4}$. The remainder of this article is organized as follows: Section 2 provides a short overview of the theoretical background of Stroud's degree three GQs. Section 3 introduces the proposed MRGQ method along with an example. It also details the approach used to generate a benchmark for evaluating the quality of the approximations made using GQ and the proposed MRGQ method. Section 4 provides an overview of the simulation models applied to compare the results between the MRGQ and GQ methods. Section 5 evaluates the approximation results generated by GQ and MRGQ by comparing them against an LHS benchmark. Sections 6 and 7 offer a discussion and some concluding remarks with respect to the potential of the MRGQ method.

\section{Theoretical background: Stroud's Gaussian quadratures}

The method presented in this section refers to the degree three quadrature formulae by Stroud (1957), aiming to obtain results with a certain degree of accuracy using the fewest possible points. Stroud's (1957, p. 259) theorem states the following:

As a necessary and sufficient condition in which $2 \mathrm{n}$ points $v_{1}, \ldots, v_{n}$ and $-v_{1}, \ldots,-v_{n}$ form an equally weighted numerical integration formula of degree three for a symmetrical region $R$, these points form the vertices of a $Q_{n}$ whose centroid coincides with the centroid of the region and lie on an $n$ sphere of radius $r=\sqrt{n I_{2} / I_{0}}$.

Here, $Q_{n}$ is a regular, $n$-dimensional generalized octahedron being integrated into an $n$-sphere, $I_{0}$ is the volume of $R$, and $I_{2}$ is the integral of the square of any variable over region $R$. Region $R$ is symmetric in the sense of Stroud's theorem if it is invariant under the group of automorphisms of an $n$-cube (Stroud 1957, p. 257). Figure 1 is a graphical representation of the theorem, which indicates that, to obtain an $n$-dimensional GQ formula of degree three for an $n$-dimensional cube, we must use $2 n$ points, which are the vertices of a regular $n$-octagon (points $1-6$ in Figure 1), the centroid of which is the centroid of the cube. Because we have $2 n$ quadrature points, which are

\footnotetext{
${ }^{4}$ The cost or efficiency of a method is usually assessed by the number of iterations required to obtain statistically robust results (Razavi and Gupta 2016a). This is particularly relevant for computationally demanding simulation models. This is the case, for example, for models with global coverage, such as the CAPRI model (Britz and Witzke 2014) and the EPIC-IIASA model (Balkovič et al. 2014), recursive-dynamic models, such as the Aglink-Cosimo model (OECD/FAO 2015), and gridded models, such as GLOBIOM (Havlík et al. 2011; Havlík et al. 2014), which can produce results on a $10 \mathrm{~km} \times 10 \mathrm{~km}$ grid level. These models comprise hundreds of thousands of variables and equations and hence require a large computational capacity for multiple solutions.
} 
supposed to be equally weighted, each weight must equal $1 / 2 n$. If these conditions are met, an approximation with an accuracy of degree three can be obtained.

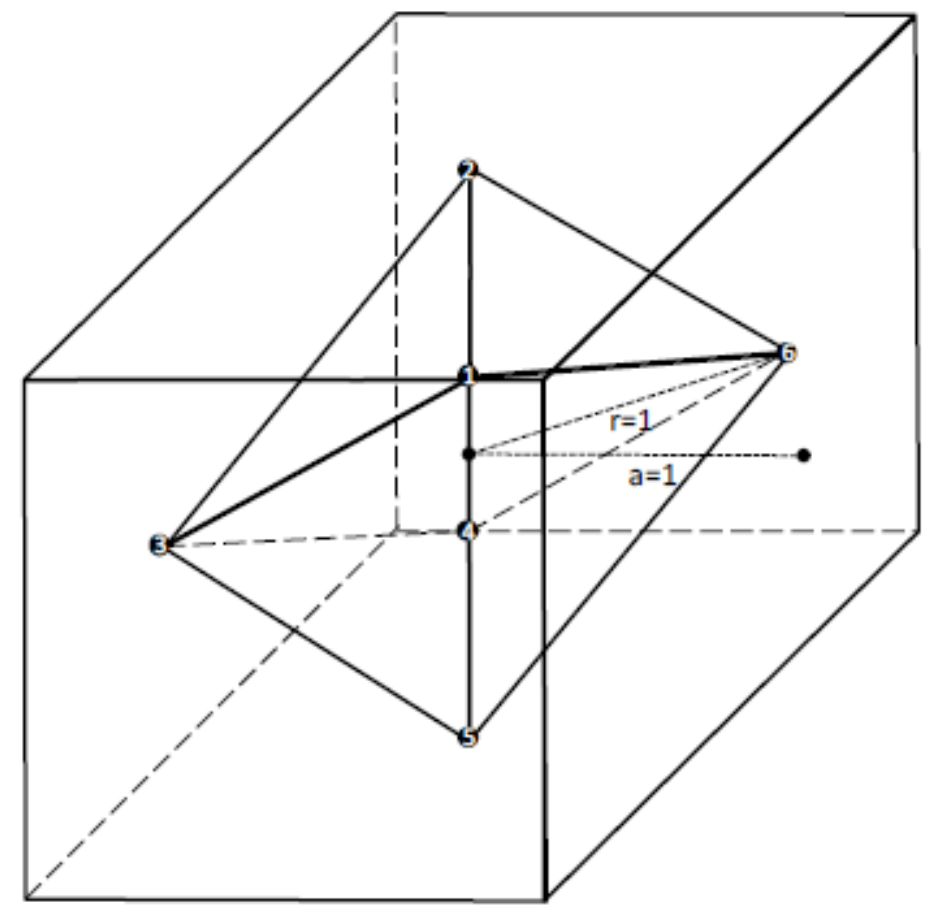

Figure 1. Graphical representation of Stroud's theorem for degree three quadrature formulae.

Notation: a, half of the side length of the cube; $r$, the radius of the n-octahedron

Source: Artavia et al. (2015)

Stroud, however, encountered a problem, in that whenever the dimensionality is greater than three, the vertices fall outside of the integration region, yielding unusable formulae. This problem can be observed in the calculation below, which is adopted from Artavia et al. (2015).

The volume of an $n$-cube $\left(C^{n}\right)$ with vertices $( \pm \mathrm{a}, \pm \mathrm{a}, \ldots, \pm \mathrm{a})$ can be obtained as follows:

(5) $I_{0}=\int_{C^{n}} x_{i}^{0} d \vec{x}=(2 a)^{n}$.

The integral of the square of any variable over this region is

(6) $I_{2}=\int_{C^{n}} x_{i}^{2} d \vec{x}=\int_{C^{n-1}} d \tilde{\vec{x}} \int_{[-a,+a]} x_{i}^{2} d x_{i}=(2 a)^{n-1}\left[\frac{1}{3} x_{i}^{3}\right]_{-a}^{+a}=(2 a)^{n-1} \frac{2}{3} a^{3}=\frac{2^{n}}{3} a^{n+2}$.

Here, $\tilde{\vec{x}} \in R^{n-1}$ is the vector $\vec{x}$ with the coordinate $x_{i}$ omitted. This yields the radius of the octahedron: 
(7) $r=\sqrt{n \frac{I_{2}}{I_{0}}}=\sqrt{n\left(\frac{2^{n}}{3} a^{n+2} / 2^{n} a^{n}\right)}=\sqrt{n \frac{a^{2}}{3}}=a \sqrt{\frac{n}{3}}$.

In the case presented in Figure 1, we deal with a three-dimensional cube with vertices $( \pm 1, \pm 1, \pm 1)$, and for $n=3$, we obtain $r=1$. However, note that, for $n>3$, we have $r>a$, and thus the vertices of the $n$-octahedron lie outside the $n$-cube.

As a solution to this problem, Stroud (1957) suggested the following formula to rotate the octahedron and bring the quadrature points back into the integration region. For $k=1, \ldots, 2 n$, let $\Gamma_{k}$ denote the quadrature point $\left(\gamma_{k, 1}, \gamma_{k, 2}, \ldots, \gamma_{k, n}\right)$, where

(8) $\gamma_{k, 2 j-1}=\sqrt{\frac{2}{3}} \cos \left(\frac{(2 j-1) k \pi}{n}\right)$,

(9) $\gamma_{k, 2 j}=\sqrt{\frac{2}{3}} \sin \left(\frac{(2 j-1) k \pi}{n}\right)$,

for $j=1, \ldots,[\mathrm{n} / 2]$, where $[n / 2]$ is the greatest integer not exceeding $n / 2$. In addition, if $n$ is odd, (10) $\gamma_{k, n}=\frac{(-1)^{k}}{\sqrt{3}}$

The quadrature points generated by these formulae fulfill the three prerequisites mentioned above. Arndt (1996) adapted Stroud's formulae for integrals over all $\mathrm{R}^{\mathrm{n}}$ (Euclidean space) with the multivariate standard normal distribution as a weight function. Arndt's formulae are Stroud points multiplied by $\sqrt{3}$, which is derived from the fact that the value of the radius $\left(r=\sqrt{n \frac{I_{2}}{I_{0}}}\right)$ changes as follows:

$$
I_{0}=\int_{R^{n}} x_{i}^{0} \frac{1}{(2 \pi)^{n / 2}} e^{-\frac{\|\vec{x}\|^{2}}{2}} d \vec{x}=(1)^{n}=1
$$

$$
I_{2}=\int_{R^{n}} x_{i}^{2} \frac{1}{(2 \pi)^{n / 2}} e^{-\frac{\|\vec{x}\|^{2}}{2}} d \vec{x}=\int_{R^{n-1}} \frac{1}{(2 \pi)^{(n-1) / 2}} e^{-\frac{\|\tilde{\vec{x}}\|^{2}}{2}} d \tilde{\vec{x}} \int_{R} x_{i}^{2} \frac{1}{(2 \pi)^{1 / 2}} e^{-\frac{x_{i}^{2}}{2}} d x_{i}=
$$

$(1)^{n-1} 1=1$.

Here, $\|\vec{x}\|$ denotes the Euclidean norm of the vector $\vec{x} \in R^{n}$, and $\tilde{\vec{x}} \in R^{n-1}$ is the vector $\vec{x}$ with the coordinate $x_{i}$ omitted; therefore, in particular, $\|\vec{x}\|^{2}=\|\tilde{\vec{x}}\|^{2}+x_{i}^{2}$.

It follows that

(13) $r=\sqrt{n \frac{I_{2}}{I_{0}}}=\sqrt{n}$. 
Therefore, Equations (8)-(10) must be adapted accordingly, and for the $k^{\text {th }}$ quadrature point $\Gamma_{k}=\left(\gamma_{k, 1}, \gamma_{k, 2}, \ldots, \gamma_{k, n}\right)$, where $\mathrm{k}=1,2, \ldots, 2 n$, we obtain the following:

(14) $\gamma_{k, 2 j-1}=\sqrt{2} \cos \left(\frac{(2 j-1) k \pi}{n}\right)$,

(15) $\gamma_{k, 2 j}=\sqrt{2} \sin \left(\frac{(2 j-1) k \pi}{n}\right)$,

for $j=1, \ldots,[\mathrm{n} / 2]$, where $[\mathrm{n} / 2]$ is the greatest integer not exceeding $n / 2$, and if $n$ is odd,

(16) $\gamma_{k, n}=(-1)^{k}$

The GQ points generated by Stroud's (1957) formulae, that is, (8)-(10), have a restricted variation around a mean of no more than $\sqrt{2 / 3} \sigma_{i}$ on each coordinate axis. Consequently, the variation of the GQ points proposed by Arndt (1996) is restricted to no more than $\sqrt{2} \sigma_{i}$ on each coordinate axis, where $\sigma_{i}$ is the standard deviation of the $i$-th uncertain input factor. This sampling interval, however, can be broadened by a desired factor using the method proposed by Preckel et al. (2011). To endow the finite distribution with the desired covariance matrix $\Sigma$, the sampling points need to be multiplied by a square matrix $A$ satisfying $\Sigma=A A^{T}$. There are several standard methods that can be used to obtain $A$ from $\Sigma$, such as eigenvalue decomposition, Cholesky factorization, or reverse Cholesky factorization (Artavia et al. 2015). Therefore, the matrix of the final quadrature points can be obtained as $G Q=\vec{\mu}+\Gamma A$, where $\vec{\mu}$ is the vector of the mean values (e.g., the base values of the input factors). In this study, we use the eigenvalue decomposition technique.

\section{Methods}

\subsection{Benchmark generation}

In the first step, we generate a reliable benchmark against which the results obtained by the proposed MRGQ method are compared. We use the well-established LHS technique and systematically determine a sufficient sample size for each model. To this end, we solve each model using the LHS technique with a converged sample size, that is, by following the convergence evaluation method suggested by Yang (2011), we solve the model with a small sample size and gradually increase it ${ }^{5}$. We observe the behavior of the coefficients of variation (CVs) of two

\footnotetext{
${ }^{5}$ For all models, we use sample sizes of 1,000 and 2,000 as well as further increases in sample sizes in increments of 2,000. Depending on the complexity of the model, we use sample sizes smaller than 1,000 as ad hoc choices.
} 
variables: the total production of each crop for which the productivity was shocked and the respective price levels. These two variables are the most relevant for simulated shocks that depict model uncertainty. In general, the variables most relevant to the respective study should be selected. The stop criterion is satisfied when the percentage of change in the results of interest, compared to the results from the previous sample size, stays within an interval of $[-1 \%, 1 \%]$. The advantages of using the CVs as an indicator are twofold: first, this measurement is dimensionless, thus facilitating a comparison, and second, it captures both the first and second moments of the data.

\section{2. $M R G Q$ method}

Artavia et al. (2015) showed that the quality of the GQ results depends strongly on the selected rotation of Stroud's octahedron. To counteract this effect, we use several families of GQ points generated from different random rotations of Stroud's octahedron. To this end, we randomly choose $k$ of $n$ ! possible permutations in the $n$ coordinates. Owing to the structure of Stroud's matrix, the easiest way to introduce random rotations is by randomly permuting the rows of Stroud's matrix. This is achieved by multiplying the matrix from the left by a permutation matrix, that is, a matrix containing a single one in each row and column and zeros everywhere else. Using $k$ permutations increases the number of quadrature points by a factor of $k$, and at the same time, considerably improves the quality of the output, as will be shown in the results section.

Following the insights of Artavia et al. (2015), we investigate how the initial position of Stroud's octahedron, from where we start the rotation, affects the final results of the GQ approximation. We generate ten series of quadratures through ten random rotations of the octahedron for the GLOBIOM model, and 20 series from 20 random rotations for each of the other two models. Note that each series contains only $2 n$ points, where $n$ is the number of uncertain variables. The number of random rotations is selected arbitrarily, considering the available computational capacities. After solving the models with the quadrature points generated by each individual rotation, we also solve them using the MRGQ method.

To evaluate the quality of the results of the MRGQ method, we compare them to the previously generated LHS benchmark. In the case of the dynamic computable general equilibrium (CGE), we follow the approach of Arndt and Thurlow (2015) and observe only the final 5-year average of the 
results, assuming that doing so will allow us to capture the cumulative effects of the uncertain input factors from previous time periods. The MRGQ method is implemented in four steps:

Step 1: Calculating the Stroud matrix

The first step is to generate a Stroud matrix for the joint standard normal distribution using Equations (14)-(16). For example, in the case of a three-dimensional problem, the Stroud matrix will have the following form:

$$
\Gamma=\left[\begin{array}{cccccc}
\frac{1}{\sqrt{2}} & -\frac{1}{\sqrt{2}} & -\sqrt{2} & -\frac{1}{\sqrt{2}} & \frac{1}{\sqrt{2}} & \sqrt{2} \\
\sqrt{\frac{3}{2}} & \sqrt{\frac{3}{2}} & 0 & -\sqrt{\frac{3}{2}} & -\sqrt{\frac{3}{2}} & 0 \\
-1 & 1 & -1 & 1 & -1 & 1
\end{array}\right] .
$$

Step 2: Transforming the covariance matrix

To incorporate the desired covariance structure and the base values into the Stroud matrix, the first step is to derive a covariance matrix of the uncertain input factors. For example, in this study, the covariance matrix is derived from historical data using the methodologies of Burrell and Nii-Naate (2013) and Araujo-Enciso et al. (2017). As an example, let us consider the following covariance matrix $\Sigma$ and the vector $\vec{\mu}$ of the base values, which in this case are randomly generated for demonstration only:

$$
\Sigma=\left[\begin{array}{ccc}
0.289558 & 0.246504 & -0.583676 \\
0.246504 & 1.430970 & 0.215241 \\
-0.583676 & 0.215241 & 1.699880
\end{array}\right], \vec{\mu}=\left[\begin{array}{c}
1.46798 \\
7.88187 \\
5.59115
\end{array}\right]
$$

As described in Section 2, to endow a finite distribution with the desired covariance matrix $\Sigma$, we need to multiply Stroud's matrix, generated in Step 1, by a square matrix $A$ satisfying $\Sigma=A A^{T}$. To obtain the square matrix $A$, we apply the diagonalization method according to the following:

(17) $\Sigma=U D U^{T}=(U \sqrt{D})\left(\sqrt{D} U^{T}\right)=A A^{T}$,

where $U$ is the matrix of the eigenvectors of $\Sigma$, and $D$ is the diagonal matrix of the eigenvalues of $\Sigma$. From the example above, we obtain the following: 


$$
D=\left[\begin{array}{ccc}
1.94088 & 0 & 0 \\
0 & 1.46611 & 0 \\
0 & 0 & 0.0134251
\end{array}\right], \quad U=\left[\begin{array}{ccc}
-0.289240 & 0.281216 & 0.915018 \\
0.250202 & 0.944856 & -0.211297 \\
0.923980 & -0.167823 & 0.343651
\end{array}\right]
$$

Solving Equation (17) yields the following:

$$
\begin{gathered}
A=U \sqrt{D}=\left[\begin{array}{ccc}
-0.289240 & 0.281216 & 0.915018 \\
0.250202 & 0.944856 & -0.211297 \\
0.923980 & -0.167823 & 0.343651
\end{array}\right] \times\left[\begin{array}{ccc}
1.39315 & 0 & 0 \\
0 & 1.21083 & 0 \\
0 & 0 & 0.115867
\end{array}\right] \\
=\left[\begin{array}{ccc}
-0.402957 & 0.340505 & 0.106020 \\
0.348570 & 1.144060 & -0.024482 \\
1.287250 & -0.203205 & 0.039818
\end{array}\right] .
\end{gathered}
$$

Step 3: Incorporating the covariance structure into the Stroud matrix

The GQ points can now be generated using the equation below:

$$
\begin{aligned}
& G Q=A \Gamma+\vec{\mu}[1 \ldots 1] \\
& =\left[\begin{array}{ccc}
-0.402957 & 0.340505 & 0.10602 \\
0.34857 & 1.14406 & -0.0244823 \\
1.28725 & -0.203205 & 0.0398178
\end{array}\right] \times\left[\begin{array}{cccccc}
\frac{1}{\sqrt{2}} & -\frac{1}{\sqrt{2}} & -\sqrt{2} & -\frac{1}{\sqrt{2}} & \frac{1}{\sqrt{2}} & \sqrt{2} \\
\sqrt{\frac{3}{2}} & \sqrt{\frac{3}{2}} & 0 & -\sqrt{\frac{3}{2}} & -\sqrt{\frac{3}{2}} & 0 \\
-1 & 1 & -1 & 1 & -1 & 1
\end{array}\right]+ \\
& {\left[\begin{array}{c}
1.46798 \\
7.88187 \\
5.59115
\end{array}\right] \times\left[\begin{array}{cccccc}
1 & 1 & 1 & 1 & 1 & 1
\end{array}\right]} \\
& =\left[\begin{array}{cccccccc}
1.49406 & 2.27597 & 1.93183 & 1.44191 & 0.659999 & 1.00414 \\
9.55401 & 9.0121 & 7.4134 & 6.20973 & 6.75165 & 8.35034 \\
6.21268 & 4.47188 & 3.73089 & 4.96962 & 6.71043 & 7.45141
\end{array}\right] .
\end{aligned}
$$

As can be seen from the final matrix obtained by Equation (19), the required sample size is equal to $2 n$, where $n$ is the number of uncertain input factors.

Step 4: Generating the $M R G Q$ points 
To perform a random rotation of the GQ, we apply a randomly generated permutation matrix, e.g., $P=\left[\begin{array}{lll}0 & 1 & 0 \\ 0 & 0 & 1 \\ 1 & 0 & 0\end{array}\right]$. Thus, the randomly generated $G Q_{\text {Rand }}$ matrix is calculated as follows:

$$
\begin{aligned}
& G Q_{\text {Rand }}=A P \Gamma+\vec{\mu}[1 \ldots 1] \\
& =\left[\begin{array}{ccc}
-0.402957 & 0.340505 & 0.106020 \\
0.348570 & 1.144060 & -0.024482 \\
1.287250 & -0.203205 & 0.039818
\end{array}\right] \times\left[\begin{array}{lll}
0 & 1 & 0 \\
0 & 0 & 1 \\
1 & 0 & 0
\end{array}\right] \times\left[\begin{array}{cccccc}
\frac{1}{\sqrt{2}} & -\frac{1}{\sqrt{2}} & -\sqrt{2} & -\frac{1}{\sqrt{2}} & \frac{1}{\sqrt{2}} & \sqrt{2} \\
\sqrt{\frac{3}{2}} & \sqrt{\frac{3}{2}} & 0 & -\sqrt{\frac{3}{2}} & -\sqrt{\frac{3}{2}} & 0 \\
-1 & 1 & -1 & 1 & -1 & 1
\end{array}\right] \\
& +\left[\begin{array}{c}
1.46798 \\
7.88187 \\
5.59115
\end{array}\right] \times\left[\begin{array}{llllll}
1 & 1 & 1 & 1 & 1 & 1
\end{array}\right] \\
& =\left[\begin{array}{llllll}
0.70893 & 1.24000 & 0.97754 & 2.22704 & 1.69597 & 1.95842 \\
7.14741 & 9.47015 & 6.77244 & 8.61633 & 6.29359 & 8.99131 \\
7.39906 & 6.93634 & 5.73805 & 3.78324 & 4.42460 & 5.44426
\end{array}\right] .
\end{aligned}
$$

This indicates that the application of the permutation matrix yields extremely different GQ points. Finally, to obtain the matrix of MRGQ points, we need to combine the GQ matrices generated by all different rotations:

$M R G Q=\left[\begin{array}{cccccccccccc}1.49406 & 2.27597 & 1.93183 & 1.44191 & 0.659999 & 1.00414 & 0.70893 & 1.24000 & 0.97754 & 2.22704 & 1.69597 & 1.95842 \\ 9.55401 & 9.0121 & 7.4134 & 6.20973 & 6.75165 & 8.35034 & 7.14741 & 9.47015 & 6.77244 & 8.61633 & 6.29359 & 8.99131 \\ 6.21268 & 4.47188 & 3.73089 & 4.96962 & 6.71043 & 7.45141 & 7.39906 & 6.93634 & 5.73805 & 3.78324 & 4.42460 & 5.44426\end{array}\right]$

Working separately with both GQ families generated above, and taking the average yields the same result as taking the union of the two families and adjusting the weights accordingly. In this case, the weights are equal to $1 / 2 \cdot 1 / 6=1 / 12$.

\section{Simulation models and data}

The MRGQ approach is tested using three different simulation models covering environmental dimensions such as land-use and weather-driven yields, i.e., a comparative-static, single-country CGE model based on the static applied general equilibrium model, ver. 2 (STAGE2) (McDonald and Thierfelder 2015), and extended for and applied to Bhutan (Feuerbacher et al. 2018) (called 
static CGE throughout this article); GLOBIOM (Havlík et al. 2011; Havlík et al. 2014), a global partial equilibrium model of the agricultural and forestry sectors; and a multi-sector recursivedynamic CGE model for the Sudan (Diao and Thurlow 2012) (called dynamic CGE throughout this article). All models are programmed using the General Algebraic Modeling System.

In all models, we simulate the uncertainty of the crop yields resulting from weather and other environmental factors (such as the prevalence of disease), which constitutes a major determinant of agricultural price volatility. For this purpose, we use historical data from agricultural databases (FAOSTAT 2018; ICRISAT 2018) and national institutions (MoAF 2016). Following Burrell and Nii-Naate (2013), we separate the uncertainty components from the historical crop yield data as deviations from the estimated trends for crops with sufficient data availability. Subsequently, uncertain input factors are generated. For example, let $y_{c, y}$ be the observed yield of crop $c$ in year $y$, where $c=(1,2, \ldots, n)$ and $y=(1,2, \ldots, m)$, and $\hat{y}_{c, y}$ be the estimated trend for the same crop in the same year. Thus, the uncertainty component $\left(z_{c, y}\right)$ is calculated as $z_{c, y}=y_{c, y} / \hat{y}_{c, y}-1$. Following the same procedure for all variables, we generate the matrix of uncertainty components (deviates) $Z_{c \times y}$. The covariance matrix of the derived uncertainty components is used to generate the multivariate distributions from which the uncertain input factors are drawn, as explained in Section 3. Because the expected value of these uncertainty components is equal to zero, it is irrelevant which crop yields are chosen as uncertain in this context. ${ }^{6}$

\subsection{Static $C G E$}

Static CGE is a single-country, comparative-static CGE model that uses the STAGE2 framework and has been documented extensively by McDonald and Thierfelder (2015). Static CGE extends the basic STAGE2 model to include a multi-level production structure of nested constant elasticity of substitution (CES) and Leontief fixed-coefficient technology functions. The demand system follows a two-stage linear expenditure system (LES)-CES nest, allowing for a substitution of commodities. The model extension and parameters were documented by Feuerbacher et al. (2018). The model is calibrated ${ }^{7}$ to a 2012 social accounting matrix for Bhutan (Feuerbacher et al. 2017) with multiple sectors, 10 of which are crop-producing.

\footnotetext{
${ }^{6}$ In this article, the choice of uncertain yield variables is based on data quality and availability.

${ }^{7}$ By calibration we mean that the model reproduces the observed data if no shock is applied (Howitt 1995).
} 
The model is run such that household saving rates are adjusted to meet a given level of investment. The exchange rate is flexible, and the foreign savings are fixed. Reflecting the short-term nature of the uncertain input factors, the model closures account for fixed land allocation (no land mobility across crop sectors), fixed government spending, and flexible government savings (all tax rates remain constant). The impact of the yield uncertainty is evaluated for all ten cropproducing sectors, namely paddy, maize, wheat, pulses, vegetables, potatoes, spices, apples, citrus fruits, and other fruits and nuts. The uncertainty in the crop yields is modeled by shocking the respective crop sector's total factor productivity (TFP). The changes in TFP are expressed using the variable $A D X_{a}$ for activity $a$ in the following model equation depicting production:

(21) $Q X_{a}=A D X_{a} \cdot\left(\delta_{a}^{x} \cdot Q V A_{a}^{-r h o c_{a}^{x}}+\left(1-\delta_{a}^{x}\right) \cdot Q I N T_{a}^{-r h o c_{a}^{x}}\right)^{\frac{-1}{r h o c_{a}^{x}}}$,

where $Q X_{a}$ is the output of activity $a ; \delta_{a}^{x}$ is the share parameter for the CES production function determining the aggregated number of factors used, that is, aggregated value added (QVA) and aggregated intermediates (QINT) used; and $r h o c_{a}^{x}$ is the substitution parameter. In addition, $A D X_{a}$ is endogenously determined according to the following adjustment mechanism:

(22) $A D X_{a}=\left[\left(a d x b_{a}+d a b a d x_{a}\right) \cdot A D X A D J\right]+\left(D A D X \cdot a d x 01_{a}\right)$,

where $a d x b$ is the base value, dabadx is an absolute change in the base value, ADXADJ is a multiplicative adjustment factor, $D A D X$ is an additive adjustment factor, and $a d x 01$ is a vector consisting of zeros and non-zeros used to scale the additive adjustment factor. The uncertainty component $($ randa) is added to Equation (22) as follows:

(23) $A D X_{a}=\left(1+\operatorname{rand}_{a}\right) \cdot\left[\left[\left(a d x b_{a}+\operatorname{dabadx}_{a}\right) \cdot A D X A D J\right]+\left(D A D X \cdot a d x 01_{a}\right)\right]$.

\subsection{GLOBIOM}

GLOBIOM is a bottom-up, recursive-dynamic partial equilibrium model with global coverage, integrating the agricultural, bioenergy, and forestry sectors (Havlík et al. 2011; Havlík et al. 2014). It is a linear programming model with a spatial equilibrium approach (Takayama and Judge 1971). The market equilibrium for agricultural and forestry products is computed based on a welfaremaximizing objective function subject to resource, technology, demand, and policy constraints. The model version applied in this study covers 31 regions globally and considers the 18 most important crops in terms of globally harvested quantities. Because this version of the model requires a large computational capacity, we use it in a comparative static framework, starting from 
a fixed 2010 solution and solving the model for only one time step (2020). We analyze the yield uncertainties of groundnuts, maize, rice, soybeans, and sugarcane grown in Indonesia, and of barley, groundnuts, sorghum, potatoes, dry beans, rice, wheat, sugarcane, maize, soybeans, cassava, and sweet potatoes grown in Brazil. In GLOBIOM, at the national level, land-use data are based on FAOSTAT statistics, which are spatially allocated using data from the spatial production allocation model (SPAM) (You and Wood 2006). Production technologies, as indicated by SPAM data, are specified through Leontief production functions. Four different management systems (irrigated-high-input, rainfed-high-input, rainfed-low-input, and subsistence) are simulated using EPIC, a biophysical-process-based crop model (Williams 1995; Izaurralde et al. 2006), and fitted to the national averages of FAOSTAT yield data for the years 1998-2002. Over the course of a particular scenario, yields react through changes in the management system, spatial reallocations, or exogenous components representing technical change. For our analysis, uncertain yield shocks are applied as exogenous shifters in the same manner as shown in Equation (23) for all management systems.

\subsection{Dynamic $C G E$}

Dynamic CGE is an economy-wide, recursive-dynamic CGE model (Diao and Thurlow 2012) linked to the IMPACT modeling system (Robinson et al. 2015). The model is calibrated to the most recent social accounting matrix for the Sudan with multiple sectors, 26 of which are crop-

producing (Siddig et al. 2018). The demand for the primary factors is governed by the CES functions, whereas the intermediate input demand is determined by the Leontief fixed-coefficient technology function. As in static CGE, we assume government savings to be flexible and all tax rates to be fixed. For the external balance, a flexible exchange rate is chosen, and the foreign savings are fixed. Finally, for the saving-investment identity, a fixed share of investment in terms of the absolute absorption is assumed, whereas household saving rates are endogenously adjusted in a uniform way to generate the necessary funds.

In the context of dynamic CGE, the uncertainty of the following crop yields is analyzed: irrigated cotton, irrigated and mechanized rain-fed sorghum, irrigated wheat, irrigated groundnuts, mechanized rain-fed millet, and mechanized and traditional rain-fed sesame. Similar to static CGE, uncertainty components affect the TFP, as presented in Equation (23). Although the recursivedynamic framework of the model is set up to project the period of 2018-2050, considering the 
large computational requirements of the LHS approach, we conduct our study for the time interval of 2018-2025 to obtain a benchmark. As extreme weather shocks in Sudan occur in a cyclical manner (MEDP 2013), every five years on average, the uncertainty components are applied every fifth year, in this case, in 2018 and 2023.

\section{Results}

The benchmark results for each model and the results generated by the proposed MRGQ method are presented in the following subsections.

\subsection{Benchmark using the LHS method}

The comparative-static single-country CGE model represents a model category that, unlike the other two models, is characterized by relatively low computational requirements. The convergence criterion in the production quantities is satisfied at 10,000 iterations. However, the convergence criterion in the prices is reached only at 20,000 iterations, which is subsequently selected as the benchmark sample size (Figure 2).

For GLOBIOM, the number of iterations is increased to 10,000. At this point, however, the convergence criterion is not satisfied for all crops: 4 out of 17 price variables still exhibit changes slightly above the $1 \%$ threshold (1.46\% at maximum). However, given the resources required to continue increasing the number of iterations (approximately 3,000 computer-hours for 12,000 iterations), we consider the results of 10,000 iterations as a reference because the limit of the available computational capacity was reached (Figure A.1).

For dynamic CGE, we evaluate the convergence by analyzing the behavior of the mean absolute $\mathrm{CVs}$ in the growth rates of the production and prices over the projected period. The convergence criterion for the price growth rate is reached at 12,000 iterations (Figure A.2). The convergence criterion for the production growth rate is reached at 14,000 iterations, which is chosen as the benchmark for the dynamic CGE model.

In the above-mentioned figures, the model dimensionality and complexity are positively correlated with the relevance of the increasing sample size required to reach the convergence of the CVs. 


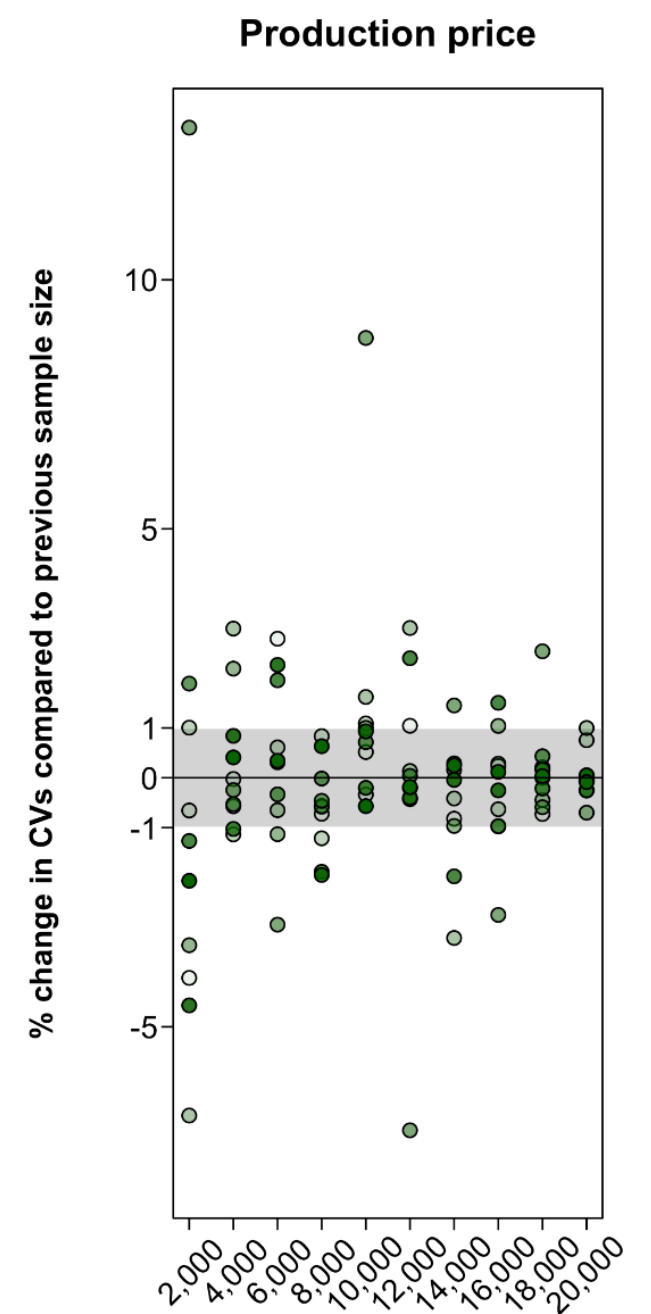

\section{Production quantity}

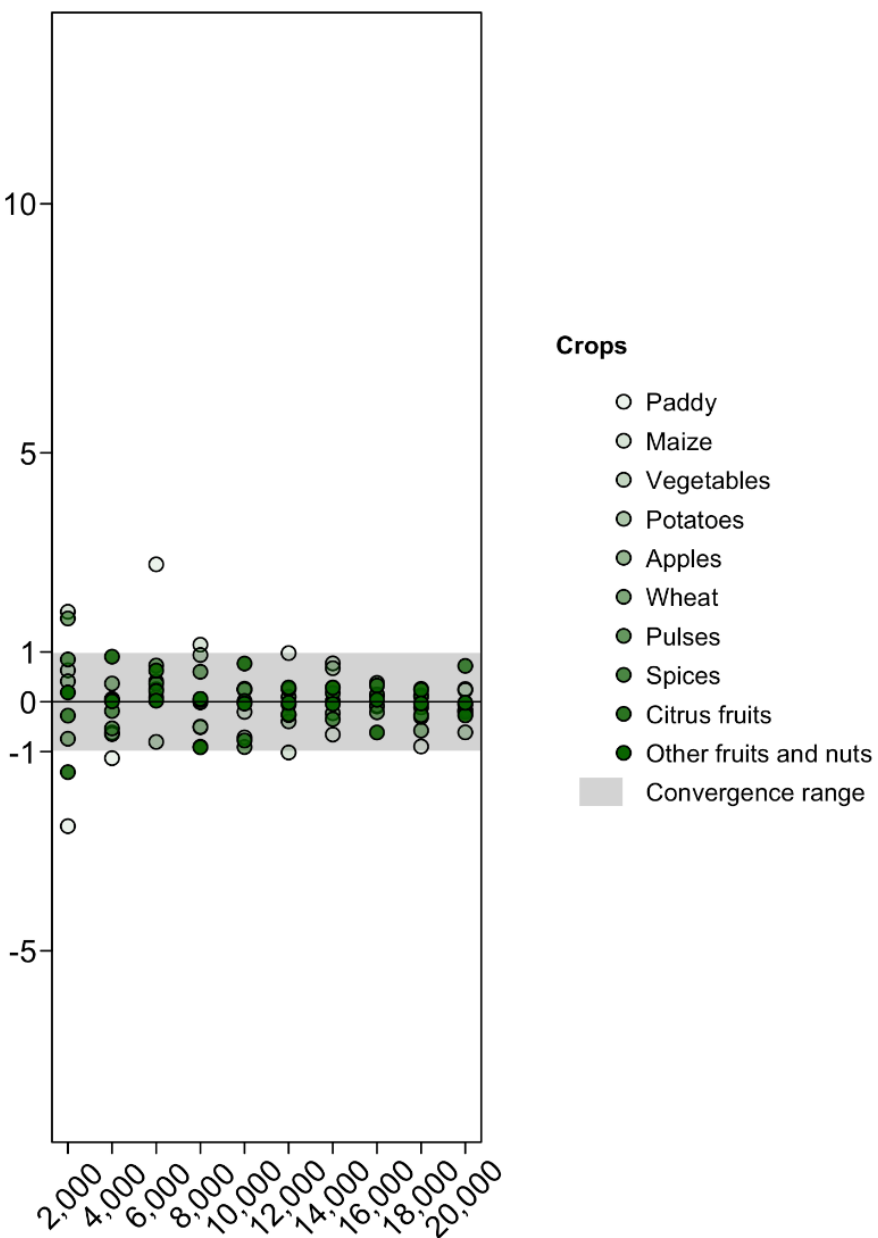

Sample size

\section{Figure 2. Convergence of the CVs of production prices and production quantities for static}

\section{CGE.}

The total factor productivity parameters of the respective crops are considered uncertain and are randomly drawn from a multivariate normal distribution using the LHS method. The starting sample size is 1,000. The sample size is gradually increased until the percentage of changes in the results compared with those obtained from the previous sample size remain within $\mathrm{a} \pm 1 \%$ range. 


\section{2. $M R G Q$ results}

Example results from each model are presented in Figures 3-5 as percent deviations from the benchmark results derived using the LHS method (for the complete results, see Figures B.1-B.3). First, the bars demonstrate that, depending on the rotations of Stroud's octahedron, the generated quadrature points lead to different levels of quality compared to the benchmark results. The largest deviations in the CVs of the production and prices from the benchmark, presented in Figures 3-5, are $-10 \%$ and $+10 \%,+11 \%$ and $-4 \%$, and $-14 \%$ and $-16 \%$, in the static CGE, GLOBIOM, and dynamic CGE models, respectively. Second, the dashed lines show that the proposed MRGQ method delivers results that are extremely close to the benchmark while also keeping the number of required iterations small compared to those required by the MC-based methods. Table 1 shows the number of iterations used by the two methods for each model and the percentage of reduction in these numbers by the proposed MRGQ method as compared to the LHS. Together with the results presented in Figures 3, 4, and 5, this shows that the MRGQ method produces high-quality results using only a fraction of the iterations required by the LHS method, and thus substantially reduces the computational effort.

Table 1. Percentage of reduction in the iterations required by the MRGQ method compared to the converged sample size iterations required by the LHS method

\begin{tabular}{lccc}
\hline & LHS & MRGQ & \% reduction \\
\hline Static CGE & 20,000 & 400 & 98.0 \\
GLOBIOM & 10,000 & 340 & 96.6 \\
Dynamic CGE & 14,000 & 280 & 98.0 \\
\hline
\end{tabular}


a) Maize production quantity

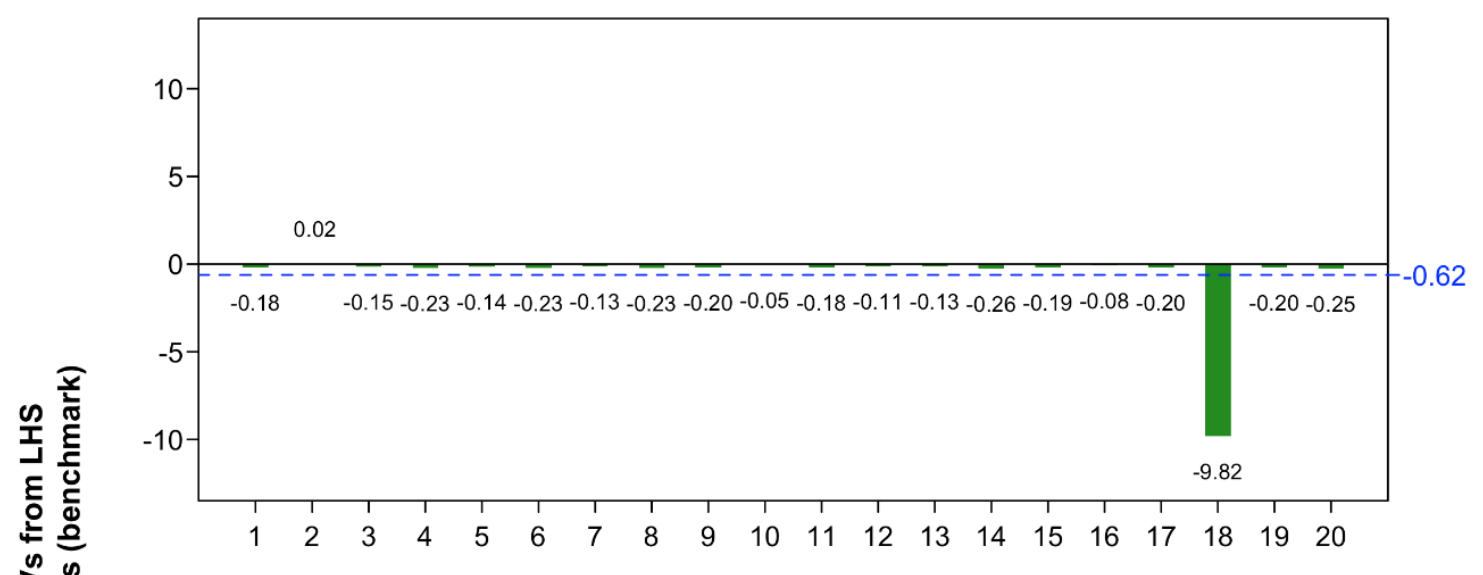

b) Paddy production price

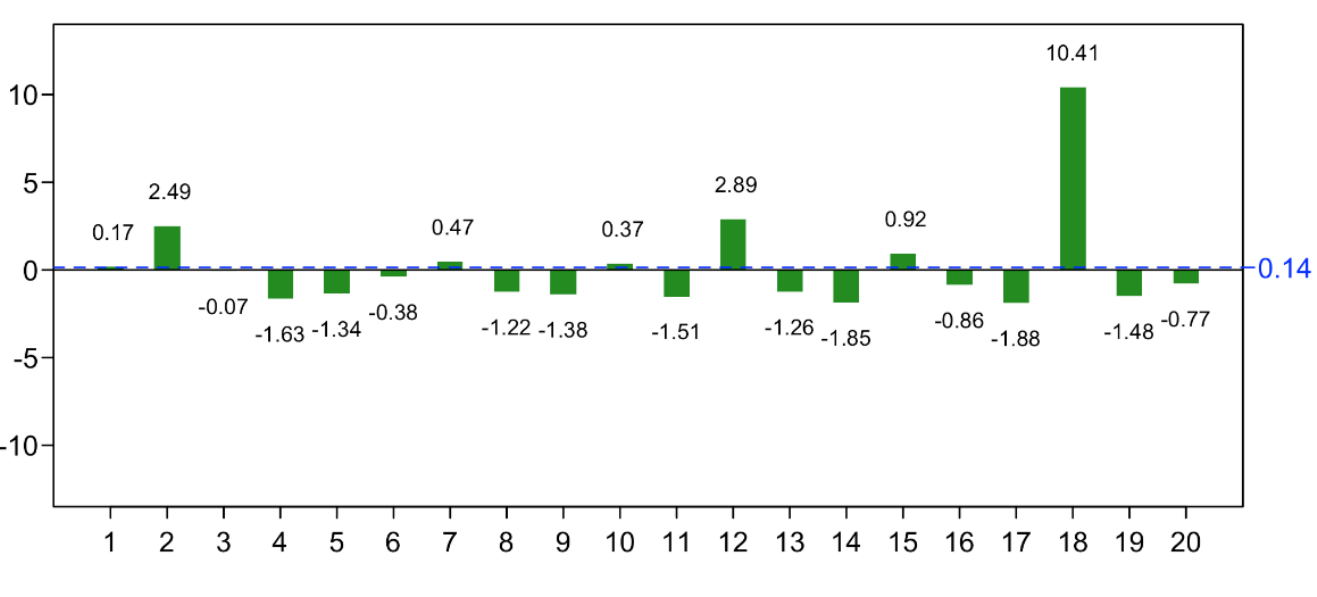

GQ rotations

Single $G Q$ rotation

- - MRGQ (average of all $G Q$ rotations)

Figure 3. Precision of single GQs and MRGQ in the static CGE model (in percent of deviation of the CVs of the results obtained by each GQ family from the benchmark).

Benchmark: LHS with 20,000 iterations. 1-20 (x-axis) are the deviations of the results obtained by 20 randomly generated GQ families; the dashed line is the result obtained by the MRGQ method (i.e., the average of these 20 GQ rotations). 
a) Groundnut production quantity in Brazil

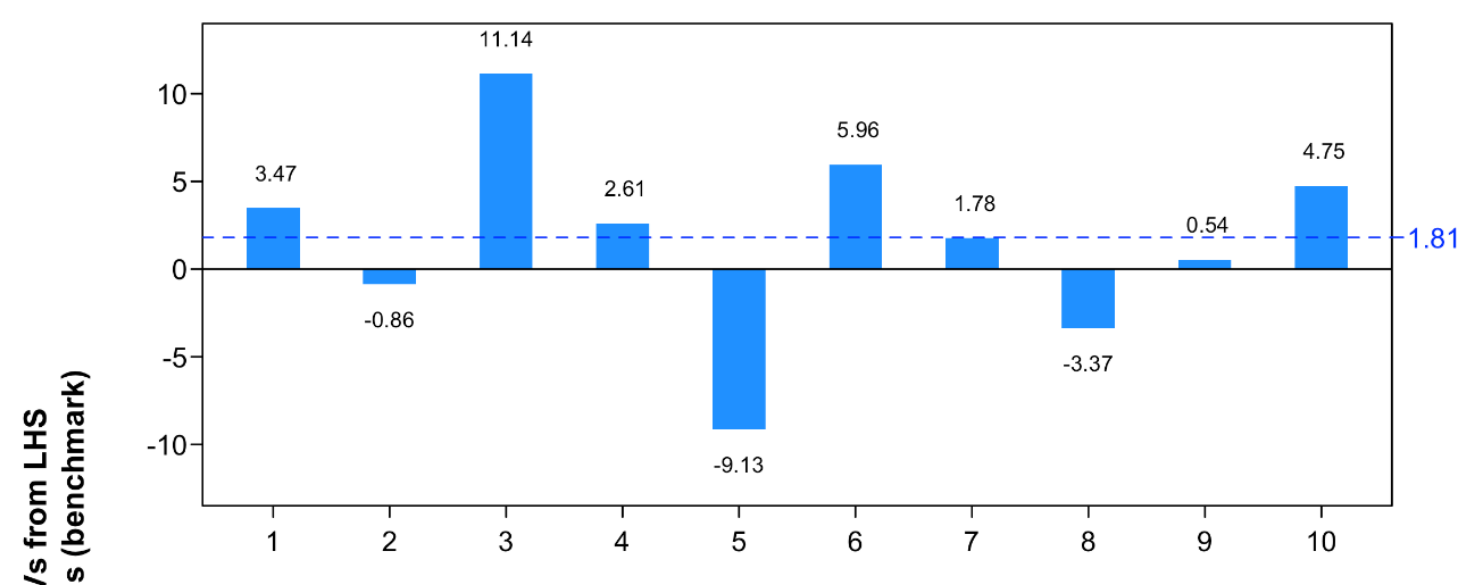

b) Dry bean production price in Brazil

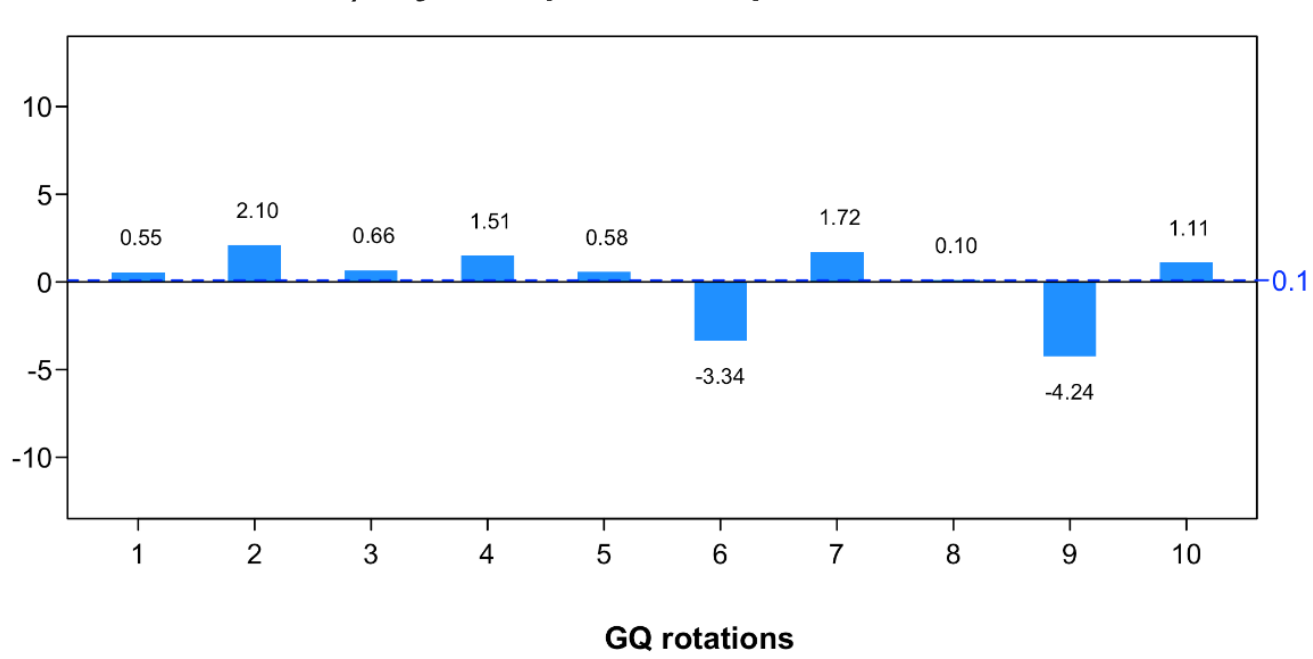

Single $G Q$ rotation

- - MRGQ (average of all $G Q$ rotations)

Figure 4. Precision of single GQs and MRGQ in the GLOBIOM model (in percent of deviation of the CVs of the results obtained by each GQ family from the benchmark).

Benchmark: LHS with 10,000 iterations. 1-10 (x-axis) are the deviations of the results obtained by 10 randomly generated GQ families; the dashed line is the result obtained by the MRGQ method (i.e., the average of these 10 GQ rotations). 


\section{a) Irrigated wheat production quantity}

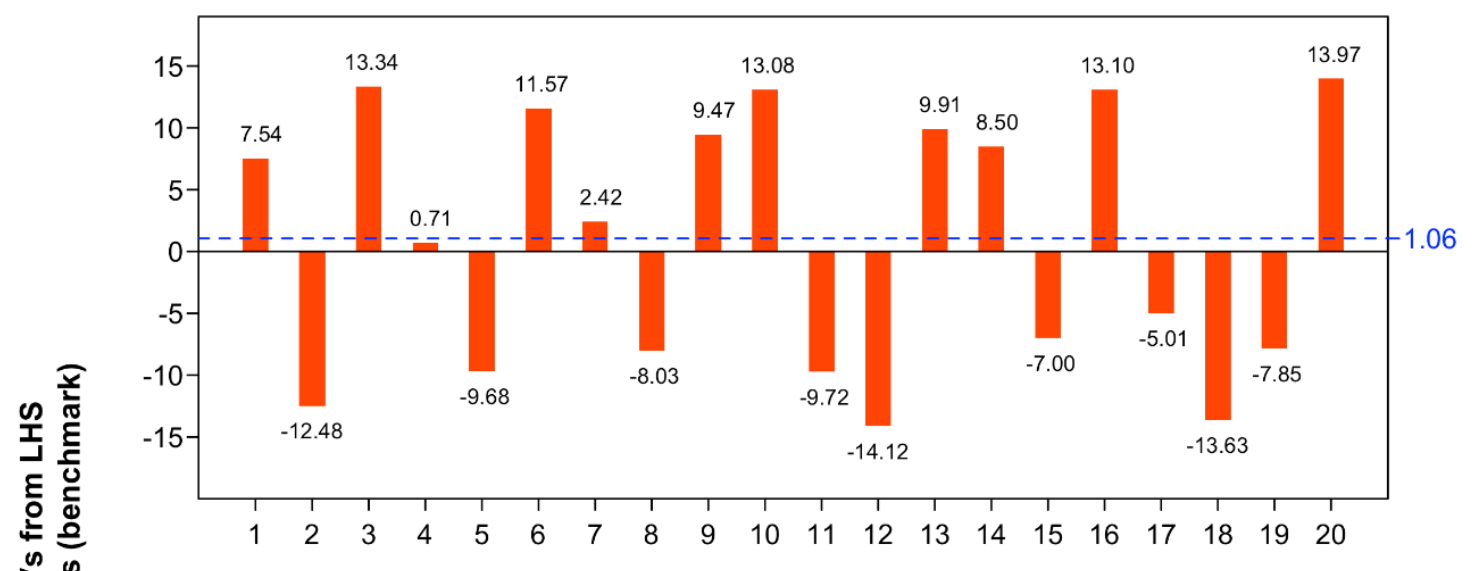

b) Mechanized rainfed sesame production price

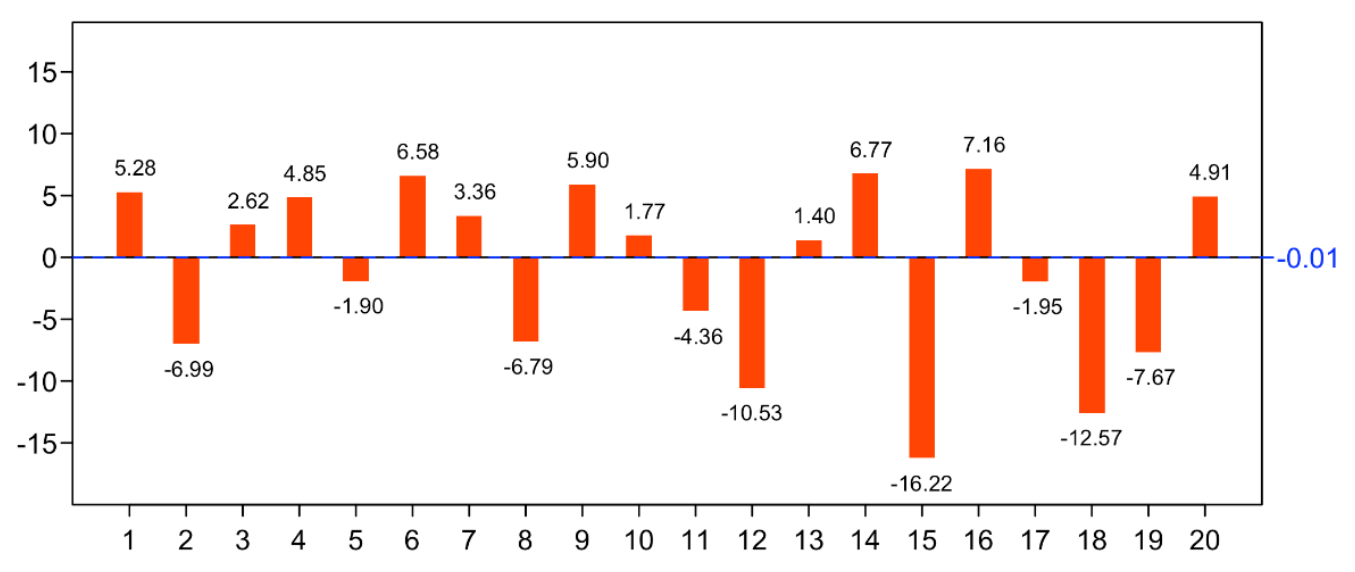

GQ rotations

Figure 5. Precision of single GQs and MRGQ in the dynamic CGE model (in percent of deviation of the CVs of the results obtained by each GQ family from the benchmark).

Benchmark: LHS with 14,000 iterations. 1-20 (x-axis) are the deviations of the results obtained by 20 randomly generated GQ families; the dashed line is the result obtained by the MRGQ method (i.e., the average of these 20 GQ rotations).

In Appendix B, we present the complete results for all crops, with yields being considered uncertain in the three models. The difference between the minimum/maximum results and the MRGQ results can be considered a measure for evaluating the improvements made by the MRGQ method as compared to the GQ method. In all three models, we observe large deviations in the 
approximated results obtained by a single GQ rotation. More specifically, in static CGE, we observe potential inaccuracies ranging from $-10 \%$ to $+1 \%$ for production and from $-28 \%$ to $+29 \%$ for prices (Figure B.1). In GLOBIOM, the inaccuracies range from $-21 \%$ to $+11 \%$ and from $-24 \%$ to $+14 \%$ for production and prices, respectively (Figure B.2). In the case of dynamic CGE, we observe inaccuracies in production within the range of $-63 \%$ to $+20 \%$. The inaccuracies in prices in dynamic CGE caused by a single GQ range from $-82 \%$ to $+35 \%$ (Figure B.3). In the vast majority of cases, we see substantial improvements in the results when applying MRGQ. The average deviations in the MRGQ result in production and price changes of $+0.04 \%$ and $-6.00 \%$ for static CGE, $-0.24 \%$ and $-1.30 \%$ for GLOBIOM, and $+0.09 \%$ and $+0.90 \%$ for dynamic CGE, respectively.

To observe the differences between the shocks produced by both methods, depicting uncertainties and their impacts on the final results, we also analyze their cumulative distribution functions (CDFs). Figure 6 presents a comparison of the CDFs of the shocks generated by the MRGQ method versus the LHS method and the resulting variables from both approaches. As the major difference between the shocks generated by these two methods, unlike the LHS method, the MRGQ method does not capture the tails of the shocks. However, this does not affect the accuracy of the approximation of the central moments of the distribution. According to two-sample t-tests and F-tests, the results obtained from the MRGQ and LHS methods shown in Figure 6 do not have statistically significant differences in means or variances at the $99 \%$ confidence level (Table 2).

Table 2. Results of two-sample t-tests and F-tests comparing the means and variances of the output distributions generated by MRGQ and LHS for the results shown in Figure 6

\begin{tabular}{llcrrl}
\hline Variable and model & Test & $\begin{array}{l}\text { Test } \\
\text { value }\end{array}$ & $\begin{array}{l}\text { Critical value } \\
\mathbf{( 9 9 . 0 0 \% )}\end{array}$ & P-value & Note \\
\hline Paddy prices generated by MRGQ & Two-Sample t-Test & 0.01 & 2.82 & 0.990 & $a$ \\
and LHS in static CGE & F-Test & 1.01 & 1.17 & 0.461 & $b$ \\
Groundnut production in Brazil by & Two-Sample t-Test & 0.05 & 2.82 & 0.960 & $a$ \\
MRGQ and LHS in GLOBIOM & F-Test & 1.04 & 1.19 & 0.300 & $b$ \\
Average mechanized rain-fed & Two-Sample t-Test & -0.23 & 2.83 & 0.820 & $a$ \\
sesame prices by MRGQ and LHS & F-Test & 1.08 & 1.23 & 0.190 & $b$ \\
in dynamic CGE & & & & & \\
\hline
\end{tabular}

Note: At a confidence level of $99 \%$ we fail to reject $\mathrm{H} 0$ in which a) means and b) variances are equal. The variables are assumed to be i.i.d., $\sim \mathrm{N}\left(0, \sigma^{2}\right)$. 
Static CGE model
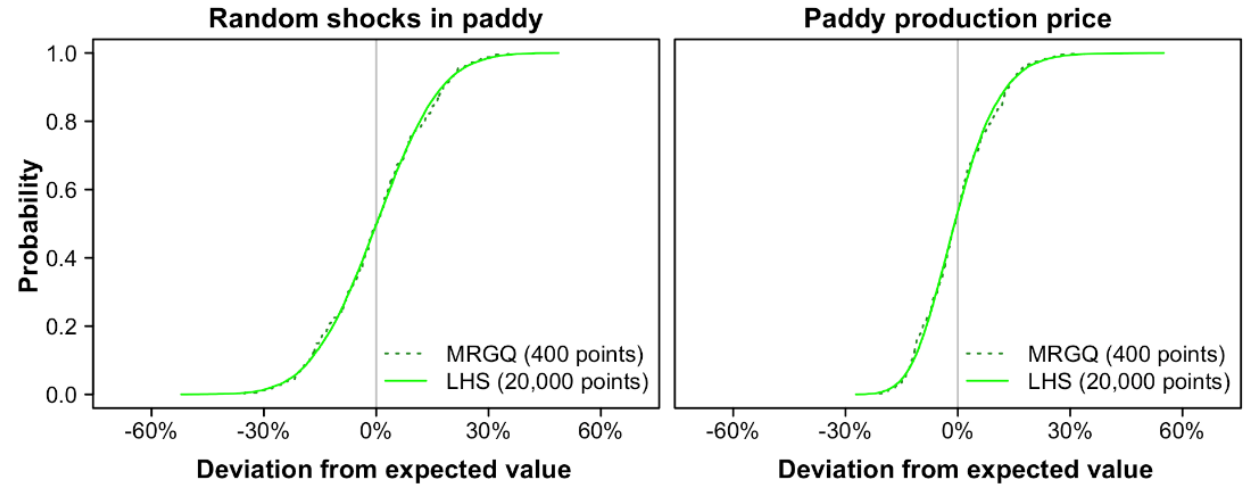

\section{GLOBIOM}

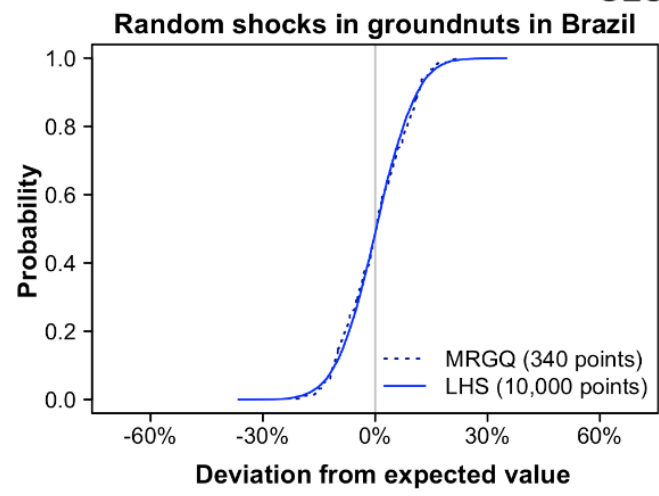

Groundnut production quantity in Brazil
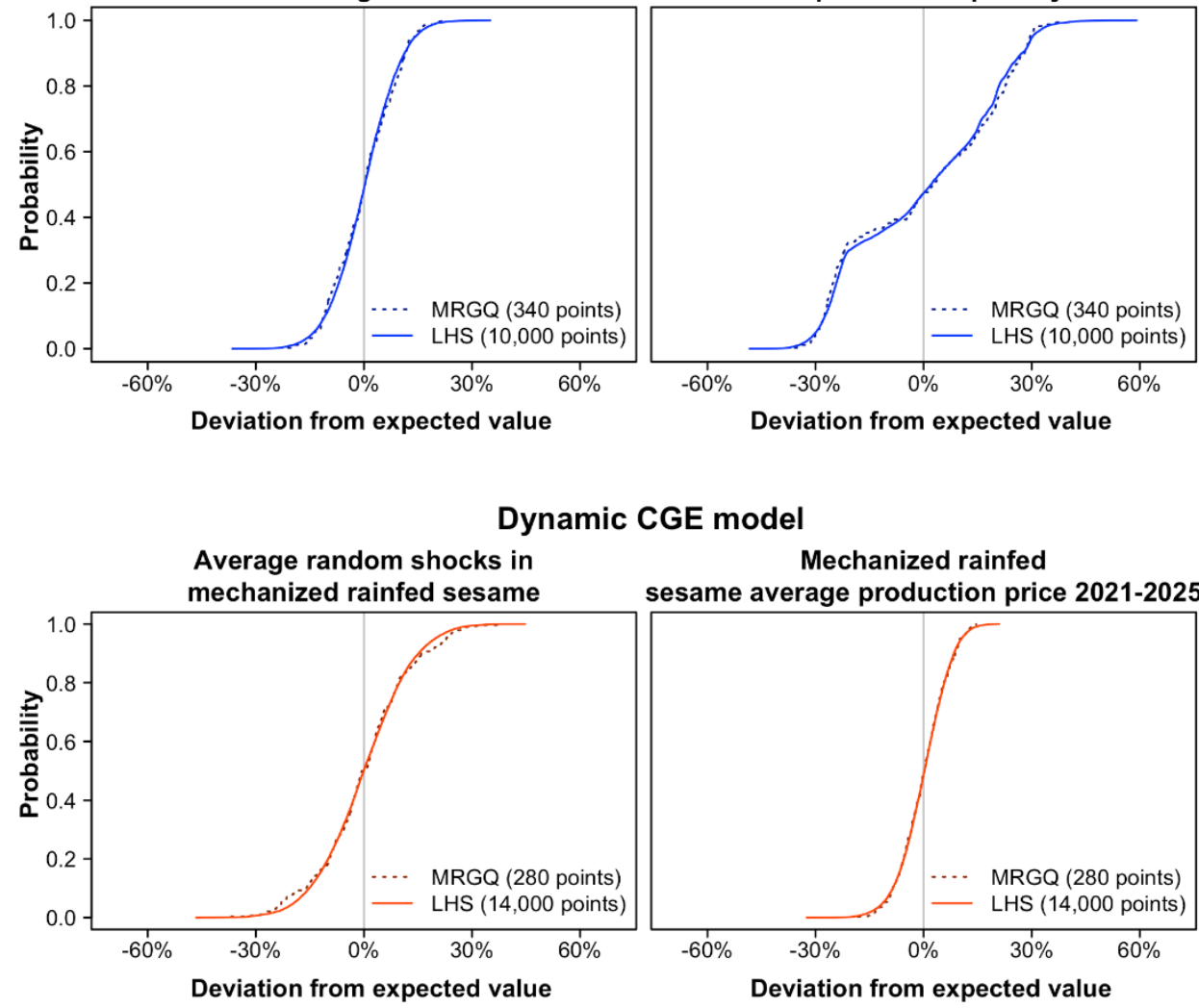

Deviation from expected value

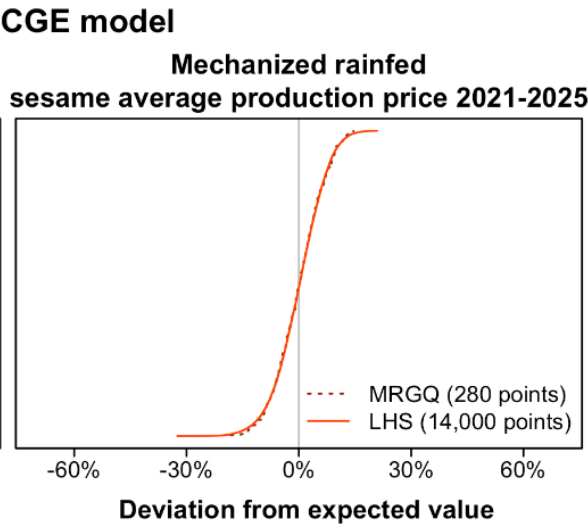

Figure 6. CDFs of the uncertain input factors and of the results obtained based on the simulation models comparing LHS and MRGQ.

The CDFs on the right-hand side indicate the results obtained from the simulation models after plugging in the uncertain input factors presented on the left-hand side. The respective number of model runs for each method is indicated in the legends. 
Table 3 presents the differences between the LHS method and the proposed novel MRGQ approach in terms of the computational and data management requirements. Owing to the modest computational requirements of static CGE, we manage to obtain a benchmark with a relatively short solving time and a small results file. However, solving the same model with the MRGQ method (using 400 iterations) is much faster, requiring only $8 \%$ of the computational time and consuming $3 \%$ of the computational space as that used by the LHS method.

Of these three models, GLOBIOM is the most computationally burdensome model to solve using the LHS approach. Solving the GLOBIOM model 10,000 times requires 2,500 computer-hours. To produce reliable results, MRGQ can solve the model using only 340 iterations, which requires only $3 \%$ of both the running time and disk space used by the LHS method. Note that the model is run for only one time step. Each additional time step increases the amount of effort proportionally. In the case of dynamic CGE, a difficulty arises from its recursive-dynamic setup. The original model is set up to project the time interval of 2018-2050. However, to obtain the benchmark results, we must shorten the interval to 2018-2025. Generating a benchmark for a single scenario requires 84 computer hours, and similar to GLOBIOM, dynamic CGE produces a results file of more than 2 GB in size. By contrast, solving the same model with the MRGQ method (using 280 iterations) requires only $6 \%$ of the running time and $9 \%$ of the disk space consumed by the LHS method, which will allow such an analysis on a single standard notebook computer. 
Table 3. Computational effort for MRGQ and LHS in three simulation models

\begin{tabular}{|c|c|c|c|c|c|c|}
\hline & \multicolumn{2}{|c|}{ LHS } & \multicolumn{4}{|c|}{ MRGQ } \\
\hline & $\begin{array}{l}\text { Size of results } \\
\text { file in } \mathrm{GB}\end{array}$ & $\begin{array}{l}\text { Model run time } \\
\text { (in computer- } \\
\text { hours) }\end{array}$ & $\begin{array}{l}\text { Size of results } \\
\text { file in } \mathrm{GB}\end{array}$ & $\begin{array}{l}\text { Reduction in the size } \\
\text { of results file } \\
\text { (percentage of } \\
\text { reduction from LHS) }\end{array}$ & $\begin{array}{l}\text { Model run time } \\
\text { (in computer- } \\
\text { hours) }\end{array}$ & $\begin{array}{l}\text { Reduction in } \\
\text { model run time } \\
\text { (percentage of } \\
\text { reduction from } \\
\text { LHS) }\end{array}$ \\
\hline Static CGE & 0.9 & 9.0 & $\begin{array}{c}0.03 \\
(20 \text { rotations })\end{array}$ & $96.7 \%$ & 0.7 & $92.2 \%$ \\
\hline GLOBIOM & 70.0 & 2,500 & $\begin{array}{c}2.36 \\
\text { (10 rotations) }\end{array}$ & $96.6 \%$ & 160.0 & $96.6 \%$ \\
\hline Dynamic CGE & 28.0 & 84.0 & $\begin{array}{c}2.60 \\
\text { (20 rotations) }\end{array}$ & $90.7 \%$ & 5.0 & $94.0 \%$ \\
\hline
\end{tabular}




\section{Discussion}

In this article, we introduce a novel approach to SSA/UA, targeting large-scale simulation models with computational restrictions. The proposed MRGQ method is based on the degree three quadrature formulae by Stroud (1957) and incorporates a novel technique to reduce the approximation error, which not only avoids computationally burdensome MC-based approaches but also offers an approximation quality that is comparable with approaches requiring extremely large sample sizes.

We test the proposed approach on three large-scale simulation models addressing agroenvironmental systems, namely a comparative static CGE model applied to Bhutan (static CGE), global partial equilibrium model (GLOBIOM), and recursive dynamic CGE model applied to the Sudan (dynamic CGE), with 10, 17, and 7 uncertain input factors, respectively. To evaluate the accuracy of the results produced by the proposed approach, we generate a benchmark using an MC-based approach, that is, LHS, with a converged sample size. To reach convergence, we follow the approach by Yang (2011), gradually increasing the sample size until the percent of deviation of the results, compared to those of the previous sample size, remain within the interval of $[-1 \%$, $+1 \%]$.

The convergence criterion is satisfied at 20,000 and 14,000 iterations for the static CGE and dynamic CGE models, respectively, whereas for GLOBIOM, we reach the available computational limit at 10,000 iterations with most of the variables satisfying the convergence criterion. These sample sizes are realized by gradually increasing the number of iterations to reach the stop criterion, thus determining the minimum required sample size in each case. For a fair comparison, the resources required to solve the models and analyze the results with smaller sample sizes would certainly have to be added to the LHS approach shown in Table 3 because such steps will be needed to determine the sample sizes required for convergence. This will substantially increase the relative advantages of the MRGQ approach. However, to the best of our knowledge, there is no established procedure for determining the starting number of iterations or steps for increasing this number. Hence, we refrained from performing such steps. It is worth noting that many studies applying MC-based approaches to SSA/UA in large-scale simulation models rarely show any convergence because of the computational burden involved (Yang 2011; Sarrazin et al. 2016). Instead, they select one sample size that fits the available computational capacities and assume that the 
approximations produced match the desired quality (Valin et al. 2015; Villoria and Preckel 2017; Mary et al. 2018).

Depending on the context of its application, there are two potential limitations of the MRGQ method. First, MRGQ does not capture the tails of the distributions because of the restricted sampling interval of Stroud's formulae (see Section 2). However, as presented in Table 2, this restriction does not limit the MRGQ method to approximate the first two central moments of the distributions with a $99 \%$ significance interval. The failure to capture the tails can be seen as both a disadvantage and an advantage. On one hand, the inability of MRGQ to depict the tails of the distributions (i.e., the effects of rare occurrences) can be viewed as a disadvantage if researchers are particularly interested in studying the impacts of extremes. In this case, we suggest implementing MRGQ along with the broader sampling approach proposed by Preckel et al. (2011). This approach allows the sampling intervals of GQ to be widened by the desired expansion factor. On the other hand, many simulation models are unable to handle large shocks to the system efficiently. Owing to technical model constraints, the systems operate far from their region of calibration, and thus far from the sound empirical foundation of the parameters. Therefore, when using MC-based approaches, researchers often truncate the distribution of the shocks (Hertel et al. 2010; OECD/FAO 2011; Burrell and Nii-Naate 2013), which may result in an inaccurate approximation of the central moments of the results. In such a case, the MRGQ approach is the most suitable method for approximating the central moments of the results without losing information about the input uncertainty.

As a second limitation, the MRGQ method is restricted to approximating symmetric distributions. The central idea of MRGQ, however, can also be applied to non-symmetric distributions. To this end, we suggest extending the MRGQ approach to depict asymmetric regions using the method developed by DeVuyst and Preckel (2007) for GQ.

\section{Conclusions}

This article describes the potential benefits of GQ as an efficient approach to UA in large-scale simulation models. It also shows the limits of traditional GQ approaches because they may generate approximations of much lower quality than those generated by traditional MC-based approaches. Therefore, we develop and test a novel MRGQ method, which overcomes the problem of insufficient accuracy of traditional GQ approaches. Applying MRGQ in three different 
simulation models reveals two distinct advantages compared to MC-based approaches. First, the MRGQ method requires a considerably smaller number of iterations when conducting a UA. This is particularly relevant for large-scale or dynamic simulation models and cases in which many variables or simulations need to be analyzed. Second, it produces highly accurate results with considerably lower computational and data management costs. The MRGQ method allows a systematic UA with high-quality outcomes in large-scale simulation models to be conducted, even in cases where MC-based approaches become infeasible because of the sample sizes and boundaries required for the computational capacity.

The demand for an efficient and robust approach to conducting UA, as offered by MRGQ, is likely to increase with the ever-expanding size and scope of the simulation models. Despite the rapid growth of computational capacities, the computational requirements in the era of "big data" require more efficient methods.

The proposed approach is successfully tested using three different simulation models integrating uncertainty in various ways. This suggests that the MRGQ method has a high potential as a resource-efficient and highly accurate means of UA in a wide range of large-scale simulation models analyzing the uncertainty of model parameters, exogenous variables, or shocks.

Although the successful application of the MRGQ method in the three case studies shows its advantages in terms of resource (computational and data management) requirements compared to MC-based approaches, open questions remain. Future research may generate a better understanding of the optimal number of random rotations required to reach a desired accuracy level, given the specific model characteristics. Moreover, research is needed to investigate the factors affecting the quality of GQ points produced by a single rotation of Stroud's octahedron.

\section{Acknowledgments}

Part of this research was funded by the German National Member Organization of the International Institute for Applied Systems Analysis (IIASA) and implemented within the framework of the Young Scientists Summer Program 2018 (YSSP). We would like to thank Zuhal Mohammed for the helpful inputs in interpreting some of the results. We would also like to thank the three anonymous reviewers for their constructive comments, which improved the article considerably. 


\section{References}

Araujo-Enciso, S. R.; Pieralli, S.; Pérez-Domínguez, I. (2017): Partial Stochastic Analysis with the Aglink-Cosimo Model: A Methodological Overview. European Commission (JRC Technical Reports). Available online at https://ec.europa.eu/jrc/en/publication/partialstochastic-analysis-aglink-cosimo-model-methodological-overview.

Arndt, C. (1996): An Introduction to Systematic Sensitivity Analysis via Gaussian Quadrature. GTAP Technical Paper (2). Available online at https://docs.lib.purdue.edu/cgi/viewcontent.cgi?referer=\&httpsredir=1\&article=1002\&contex t=gtaptp.

Arndt, C.; Fant, C.; Robinson, S.; Strzepek, K. (2015): Informed selection of future climates. In Climatic Change 130 (1), pp. 21-33. DOI: 10.1007/s10584-014-1159-3.

Arndt, C.; Hertel, T. W. (1997): Revisiting “The Fallacy of Free Trade”. In Review of International Economics 5 (2), pp. 221-229. DOI: 10.1111/1467-9396.00052.

Arndt, C.; Preckel, P. V. (2006): Efficient Survey Sampling of Households via Gaussian Quadrature. In Applied Statistics 55 (3), pp. 355-364. DOI: 10.1111/j.14679876.2006.00537.x.

Arndt, C.; Thurlow, J. (2015): Climate uncertainty and economic development: evaluating the case of Mozambique to 2050. In Climatic Change 130 (1), pp. 63-75. DOI: 10.1007/s10584014-1294-X.

Artavia, M.; Grethe, H.; Zimmermann, G. (2015): Stochastic market modeling with Gaussian Quadratures: Do rotations of Stroud's octahedron matter? In Economic Modelling 45, pp. 155-168. DOI: 10.1016/j.econmod.2014.10.017.

Balkovič, J.; van der Velde, M.; Skalský, R.; Xiong, W.; Folberth, C.; Khabarov, N. et al. (2014): Global wheat production potentials and management flexibility under the representative concentration pathways. In Global and Planetary Change 122, pp. 107-121. DOI: 10.1016/j.gloplacha.2014.08.010.

Beckman, J.; Hertel, T.; Tyner, W. (2011): Validating energy-oriented CGE models. In Energy Economics 33 (5), pp. 799-806. DOI: 10.1016/j.eneco.2011.01.005.

Britz, W.; Witzke, P. (2014): CAPRI model documentation 2014. Bonn, Germany. Available online at https://www.capri-model.org/docs/capri_documentation.pdf.

Burrell, A.; Nii-Naate, Z. (2013): Partial stochastic analysis with the European Commission's version of the AGLINK-COSIMO model. European Commission (JRC Reference Reports). DOI: $10.2791 / 87727$.

Cho, E.; Arhonditsis, G. B.; Khim, J.; Chung, S.; Heo, T. Y. (2016): Modeling metal-sediment interaction processes: Parameter sensitivity assessment and uncertainty analysis. In Environmental Modelling \& Software 80, pp. 159-174. DOI: 10.1016/j.envsoft.2016.02.026. 
Cukier, R. I.; Fortuin, C. M.; Shuler, K. E.; Petschek, A. G.; Schaibly, J. H. (1973): Study of the sensitivity of coupled reaction systems to uncertainties in rate coefficients. I Theory. In The Journal of Chemical Physics 59 (8), pp. 3873-3878. DOI: 10.1063/1.1680571.

DeVuyst, E. A.; Preckel, P. V. (2007): Gaussian cubature: A practitioner's guide. In Mathematical and Computer Modelling 45 (7-8), pp. 787-794. DOI:

10.1016/j.mcm.2006.07.021.

Diao, X.; Thurlow, J. (2012): A recursive dynamic computable general equilibrium model. In X. Diao, J. Thurlow, S. Benin, S. Fan (Eds.): Strategies and priorities for African agriculture. Economywide perspectives from country studies. Washington, D.C.: International Food Policy Research Institute (IFPRI). DOI: 10.2499/9780896291959.

Douglas-Smith, D.; Iwanaga, T.; Croke, B. F.W.; Jakeman, A. J. (2020): Certain trends in uncertainty and sensitivity analysis: An overview of software tools and techniques. In Environmental Modelling \& Software 124, p. 104588. DOI: 10.1016/j.envsoft.2019.104588.

Engels, H. (1980): Numerical Quadrature and Cubature, Computational Mathematics and Applications, London, Academic Press 1980. ISBN 0-12-238850-X. Computational Mathematics and Applications. London: Academic Press (62).

European Commission (2018): EU Agricultural Outlook for Markets and Income 2018-2030. European Commission, DG Agriculture and Rural Development, Brussels. Available online at https://ec.europa.eu/info/sites/info/files/food-farmingfisheries/farming/documents/medium-term-outlook-2018-report_en.pdf.

FAOSTAT (2018): Food and Agriculture Organization of the United Nations. FAOSTAT Statistical Database. Rome. Available online at http://www.fao.org/faostat/en/\#compare.

Feuerbacher, A.; Luckmann, J.; Boysen, O.; Zikeli, S.; Grethe, H. (2018): Is Bhutan destined for $100 \%$ organic? Assessing the economy-wide effects of a large-scale conversion policy. In PLOS ONE 13 (6). DOI: 10.1371/journal.pone.0199025.

Feuerbacher, A.; Dukpa, C.; Grethe, H. (2017): A 2012 Social Accounting Matrix (SAM) for Bhutan with a detailed representation of the agricultural sector. Humboldt-Universität zu Berlin. Berlin, Germany (94). Available online at https://www.agrar.huberlin.de/de/institut/departments/daoe/publ/wp/wp94.pdf.

Gan, Y.; Duan, Q.; Gong, W.; Tong, C.; Sun, Y.; Chu, W. et al. (2014): A comprehensive evaluation of various sensitivity analysis methods: A case study with a hydrological model. In Environmental Modelling \& Software 51, pp. 269-285. DOI: 10.1016/j.envsoft.2013.09.031.

Gouel, C.; Jean, S. (2013): Optimal Food Price Stabilization in a Small Open Developing Country. In The World Bank Economic Review 29 (1), pp. 72-101. DOI: 10.1093/wber/lht018. 
Haber, S. (1970): Numerical Evaluation of Multiple Integrals. In SIAM Review 12 (4), pp. 481522. Available online at https://www.jstor.org/stable/2028488.

Havlík, P.; Schneider, U. A.; Schmid, E.; Böttcher, H.; Fritz, S.; Skalský, R. et al. (2011): Global land-use implications of first and second generation biofuel targets. In Energy Policy 39 (10), pp. 5690-5702. DOI: 10.1016/j.enpol.2010.03.030.

Havlík, P.; Valin, H.; Herrero, M.; Obersteiner, M.; Schmid, E.; Rufino, M. C. et al. (2014): Climate change mitigation through livestock system transitions. In Proceedings of the National Academy of Sciences of the United States of America 111 (10), pp. 3709-3714. DOI: 10.1073/pnas.1308044111.

Helton, J. C.; Davis, F. J. (2003): Latin hypercube sampling and the propagation of uncertainty in analyses of complex systems. In Reliability Engineering \& System Safety 81 (1), pp. 2369. DOI: 10.1016/S0951-8320(03)00058-9.

Hertel, T. W.; Martin, W.; Leister, A. M. (2010): Potential Implications of a Special Safeguard Mechanism in the World Trade Organization: the Case of Wheat. In The World Bank Economic Review 24 (2), pp. 330-359. DOI: 10.1093/wber/lhq010.

Howitt, R. (1995). Positive Mathematical Programming. American Journal of Agricultural Economics, 77(2), 329-342. DOI:10.2307/1243543.

ICRISAT (2018): Village Dynamics in South Asia Database: Crop Yield Data. ICRISAT, New Delhi, India: Available online at http://vdsa.icrisat.ac.in/vdsa-database.aspx.

Izaurralde, R. C.; Williams, J. R.; McGill, W. B.; Rosenberg, N. J.; Quiroga Jakas, M. C. (2006): Simulating soil $\mathrm{C}$ dynamics with EPIC: Model description and testing against long-term data. In Ecological Modelling 192 (3-4), pp. 362-384. DOI: 10.1016/j.ecolmodel.2005.07.010.

Kwakkel, J. H.; Eker, S.; Pruyt, E. (2016): How Robust is a Robust Policy? Comparing Alternative Robustness Metrics for Robust Decision-Making. In Doumpos, M., Zopounidis, C., Grigoroudis, E. (Eds.): Robustness Analysis in Decision Aiding, Optimization, and Analytics. Cham: Springer International Publishing, pp. 221-237. DOI: https://doi.org/10.1007/978-3-319-33121-8_10.

Lammoglia, S. K.; Brun, F.; Quemar, T.; Moeys, J.; Barriuso, E.; Gabrielle, B.; Mamy, L. (2018): Modelling pesticides leaching in cropping systems: Effect of uncertainties in climate, agricultural practices, soil and pesticide properties. In Environmental Modelling \& Software 109, pp. 342-352. DOI: 10.1016/j.envsoft.2018.08.007.

Loucks, D. P.; van Beek, E. (2017): System Sensitivity and Uncertainty Analysis. In Loucks, D. P., van Beek, E. (Eds.): Water Resource Systems Planning and Management: An Introduction to Methods, Models, and Applications. Cham: Springer International Publishing, pp. 331374. DOI: https://doi.org/10.1007/978-3-319-44234-1_8. 
Mary, S.; Phimister, E.; Roberts, D.; Santini, F. (2018): A Monte Carlo filtering application for systematic sensitivity analysis of computable general equilibrium results. In Economic Systems Research 31 (3), pp. 404-422. DOI: 10.1080/09535314.2018.1543182.

Matott, L. S.; Babendreier, J. E.; Purucker, S. T. (2009): Evaluating uncertainty in integrated environmental models: A review of concepts and tools. In Water Resources Research 45 (6), p. 716. DOI: 10.1029/2008WR007301.

McDonald, S.; Thierfelder, K. (2015): A Static Applied General Equilibrium Model: Technical Documentation: STAGE Version 2. Model Documentation. Available online at http://www.cgemod.org.uk/stage2.pdf.

MEDP (2013): Sudan's Second National Communication under the United Nations Framework Convention on Climate Change. Ministry of Environment, Natural Resources and Physical Development (MEDP). Khartoum, Sudan. Available online at https://unfccc.int/resource/docs/natc/sudnc2.pdf.

Metropolis, N.; Ulam, S. (1949): The Monte Carlo method. In Journal of the American Statistical Association 44 (247), pp. 335-341. DOI: 10.2307/2280232.

MoAF (2016): Microdata on the Agricultural Sample Survey rounds conducted in 2012, 2013, 2014 and 2015 first and second year half. Ministry of Agriculture and Forests (MoAF). Thimphu, Bhutan.

Morris, M. D. (1991): Factorial Sampling Plans for Preliminary Computational Experiments. In Technometrics 33 (2), pp. 161-174. DOI: 10.2307/1269043.

Moss, J. E.; Binfield, J. C.R.; Zhang, L.; Patton, M.; Kim, I. S. (2010): A Stochastic Analysis of the Impact of Volatile World Agricultural Prices on European and UK Agriculture. FAPRIUK Technical Report. University of Missouri. Columbia, Missouri (108771). DOI: 10.22004/ag.econ.108771.

Norton, J. (2015): An introduction to sensitivity assessment of simulation models. In Environmental Modelling \& Software 69, pp. 166-174. DOI: 10.1016/j.envsoft.2015.03.020.

OECD/FAO (2011): Agricultural Outlook 2011-2020. OECD Publishing, Paris. DOI: 10.1787/agr_outlook-2011-en.

OECD/FAO (2015): Aglink-Cosimo model documentation. A partial equilibrium model of world agricultural markets. Available online at http://www.agri-outlook.org/about/Aglink-Cosimomodel-documentation-2015.pdf.

OECD/FAO (2017): Agricultural Outlook 2017-2026. OECD Publishing, Paris. DOI: 10.1787/agr_outlook-2017-en.

OECD/FAO (2018): Agricultural Outlook 2018-2027. OECD Publishing, Paris. DOI: 10.1787/agr_outlook-2018-en. 
O'Hagan, A. (2006): Bayesian analysis of computer code outputs: A tutorial. In Reliability Engineering \& System Safety 91 (10-11), pp. 1290-1300. DOI: 10.1016/j.ress.2005.11.025.

O’Hagan, A. (2012): Probabilistic uncertainty specification: Overview, elaboration techniques and their application to a mechanistic model of carbon flux. In Environmental Modelling \& Software 36, pp. 35-48. DOI: 10.1016/j.envsoft.2011.03.003.

Pianosi, F.; Beven, K.; Freer, J.; Hall, J. W.; Rougier, J.; Stephenson, D. B.; Wagener, T. (2016): Sensitivity analysis of environmental models: A systematic review with practical workflow. In Environmental Modelling \& Software 79, pp. 214-232. DOI: 10.1016/j.envsoft.2016.02.008.

Preckel, P. V.; Verma, M.; Hertel, T.; Martin, W. (2011): Implications of Broader Sampling Strategy in the Contest of the Special Safeguard Mechanism. Presented at the $14^{\text {th }}$ Annual Conference on Global Economic Analysis, Venice, Italy. Global Trade Analysis Project (GTAP). Department of Agricultural Economics, Purdue University, West Lafayette, IN. Available online at https://www.gtap.agecon.purdue.edu/resources/res_display.asp?RecordID=3570.

Razavi, S.; Gupta, H. V. (2016a): A new framework for comprehensive, robust, and efficient global sensitivity analysis: 1 . Theory. In Water Resources Research 52 (1), pp. 423-439. DOI: $10.1002 / 2015$ WR017558.

Razavi, S.; Gupta, H. V. (2016b): A new framework for comprehensive, robust, and efficient global sensitivity analysis: 2. Application. In Water Resources Research 52 (1), pp. 440-455. DOI: $10.1002 / 2015$ WR017559.

Razavi, S.; Sheikholeslami, R.; Gupta, H. V.; Haghnegahdar, A. (2019): VARS-TOOL: A toolbox for comprehensive, efficient, and robust sensitivity and uncertainty analysis. In Environmental Modelling \& Software 112, pp. 95-107. DOI: 10.1016/j.envsoft.2018.10.005.

Robinson, S.; Mason-D’Croz, D.; Islam, S.; Sulser, T. B.; Robertson, R.; Zhu, T. et al. (2015): The International Model for Policy Analysis of Agricultural Commodities and Trade (IMPACT). Model Description for Version 3. International Food Policy Research Institute (IFPRI). Washington, D.C. Available online at http://ebrary.ifpri.org/cdm/ref/collection/p15738coll2/id/129825.

Saltelli, A.; Ratto, M.; Andres, T.; Campolongo, F.; Cariboni, J.; Gatelli, D. et al. (2008): Global sensitivity analysis: The primer. John Wiley \& Sons, Ltd, Chichester, West Sussex.

Saltelli, A. (2002): Making best use of model evaluations to compute sensitivity indices. In Computer Physics Communications 145 (2), pp. 280-297.DOI: 10.1016/S00104655(02)00280-1.

Saltelli, A.; Aleksankina, K.; Becker, W.; Fennell, P.; Ferretti, F.; Holst, N. et al. (2019): Why so many published sensitivity analyses are false: A systematic review of sensitivity analysis practices. In Environmental Modelling \& Software 114, pp. 29-39. DOI: 10.1016/j.envsoft.2019.01.012. 
Saltelli, A.; Annoni, P. (2010): How to avoid a perfunctory sensitivity analysis. In Environmental Modelling \& Software 25 (12), pp. 1508-1517. DOI: 10.1016/j.envsoft.2010.04.012.

Saltelli, A.; Annoni, P.; Azzini, I.; Campolongo, F.; Ratto, M.; Tarantola, S. (2010): Variance based sensitivity analysis of model output. Design and estimator for the total sensitivity index. In Computer Physics Communications 181 (2), pp. 259-270. DOI: 10.1016/j.cpc.2009.09.018.

Sarrazin, F.; Pianosi, F.; Wagener, T. (2016): Global Sensitivity Analysis of environmental models: Convergence and validation. In Environmental Modelling \& Software 79, pp. 135152. DOI: $10.1016 /$ j.envsoft.2016.02.005.

Schürer, R. (2001): Parallel High-Dimensional Integration: Quasi-Monte Carlo versus Adaptive Cubature Rules. In Vassil N. Alexandrov, Jack J. Dongarra, Benjoe A. Juliano, René S. Renner, C. J. Kenneth Tan (Eds.): Computational Science - ICCS 2001. Springer, Berlin Heidelberg, pp. 1262-1271.

Schürer, R. (2003): A comparison between (quasi-)Monte Carlo and cubature rule based methods for solving high-dimensional integration problems. In Mathematics and Computers in Simulation 62 (3-6), pp. 509-517. DOI: 10.1016/S0378-4754(02)00250-1.

Sheikholeslami, R.; Razavi, S.; Gupta, H. V.; Becker, W.; Haghnegahdar, A. (2019): Global sensitivity analysis for high-dimensional problems: How to objectively group factors and measure robustness and convergence while reducing computational cost. In Environmental Modelling \& Software 111, pp. 282-299. DOI: 10.1016/j.envsoft.2018.09.002.

Siddig, K.; Elagra, S.; Grethe, H.; Mubarak, A. (2018): A post-separation Social Accounting Matrix for the Sudan (MENA RP Working Paper 8. Washington, D.C. and Cairo, Egypt: International Food Policy Research Institute (IFPRI), 8). Available online at http://ebrary.ifpri.org/cdm/ref/collection/p15738coll2/id/132312.

Song, X. M.; Zhan, C. S.; Xia, J. (2012): Integration of a statistical emulator approach with the SCE-UA method for parameter optimization of a hydrological model. In Chinese Science Bulletin 57 (26), pp. 3397-3403. DOI: 10.1007/s11434-012-5305-X.

Stanfill, B.; Mielenz, H.; Clifford, D.; Thorburn, P. (2015): Simple approach to emulating complex computer models for global sensitivity analysis. In Environmental Modelling \& Software 74, pp. 140-155. DOI: https://doi.org/10.1016/j.envsoft.2015.09.011.

Stroud, A. H. (1957): Remarks on the Disposition of Points in Numerical Integration Formulas. In Mathematical Tables and Other Aids to Computation 11 (60), pp. 257-261. DOI: $10.2307 / 2001945$.

Takayama, T.; Judge, G. G. (1971): Spatial and Temporal Price and Allocation Models. NorthHolland Publishing Company, Amsterdam, Netherlands (Contributions to economic analysis). Available online at https://books.google.de/books?id=Uau4AAAAIAAJ. 
Uusitalo, L.; Lehikoinen, A.; Helle, I.; Myrberg, K. (2015): An overview of methods to evaluate uncertainty of deterministic models in decision support. In Environmental Modelling \& Software 63, pp. 24-31. DOI: 10.1016/j.envsoft.2014.09.017.

Valenzuela, E.; Hertel, T. W.; Keeney, R.; Reimer, J. J. (2007): Assessing Global Computable General Equilibrium Model Validity Using Agricultural Price Volatility. In American Journal of Agricultural Economics 89 (2), pp. 383-397. DOI: 10.1111/j.14678276.2007.00977.x.

Valin, H.; Peters, D.; van den Berg, M.; Frank, S.; Havlik, P.; Forsell, N. et al. (2015): The land use change impact of biofuels consumed in the EU: Quantification of area and greenhouse gas impacts. Utrecht, Netherlands. Available online at http://pure.iiasa.ac.at/id/eprint/12310/.

Verma, M.; Hertel, T. W.; Valenzuela, E. (2011): Are The Poverty Effects of Trade Policies Invisible? In The World Bank Economic Review 25 (2), pp. 190-211. DOI: 10.1093/wber/lhr014.

Villoria, N. B.; Golub, A.; Byerlee, D.; Stevenson, J. (2013): Will Yield Improvements on the Forest Frontier Reduce Greenhouse Gas Emissions? A Global Analysis of Oil Palm. In American Journal of Agricultural Economics 95 (5), pp. 1301-1308. DOI: 10.1093/ajae/aat034.

Villoria, N. B.; Preckel, P. V. (2017): Gaussian Quadratures vs. Monte Carlo Experiments for Systematic Sensitivity Analysis of Computable General Equilibrium Model Results. In Economics Bulleltin 37 (1), pp. 480-487. Available online at http://www.accessecon.com/Pubs/EB/2017/Volume37/EB-17-V37-I1-P43.pdf.

Westhoff, P. C.; Brown, S.; Hart, C. (2005): When Point Estimates Miss the Point: Stochastic Modeling of WTO Restrictions. Iowa State University, Department of Economics. Available online at https://EconPapers.repec.org/RePEc:isu:genres:31341.

Williams, J. R. (1995): The EPIC Model. In V. P. Singh (Ed.): Computer models of watershed hydrology. Water Resources Publications, pp. 909-1000. ISBN: 09-183-34918.

Yang, J. (2011): Convergence and uncertainty analyses in Monte-Carlo based sensitivity analysis. In Environmental Modelling \& Software 26 (4), pp. 444-457. DOI: 10.1016/j.envsoft.2010.10.007.

You, L.; Wood, S. (2006): An entropy approach to spatial disaggregation of agricultural production. In Agricultural Systems 90 (1-3), pp. 329-347. DOI: 10.1016/j.agsy.2006.01.008.

Zhan, C. S.; Song, X. M.; Xia, J.; Tong, C. (2013): An efficient integrated approach for global sensitivity analysis of hydrological model parameters. In Environmental Modelling \& Software 41, pp. 39-52. DOI: 10.1016/j.envsoft.2012.10.009. 


\section{Appendix A}

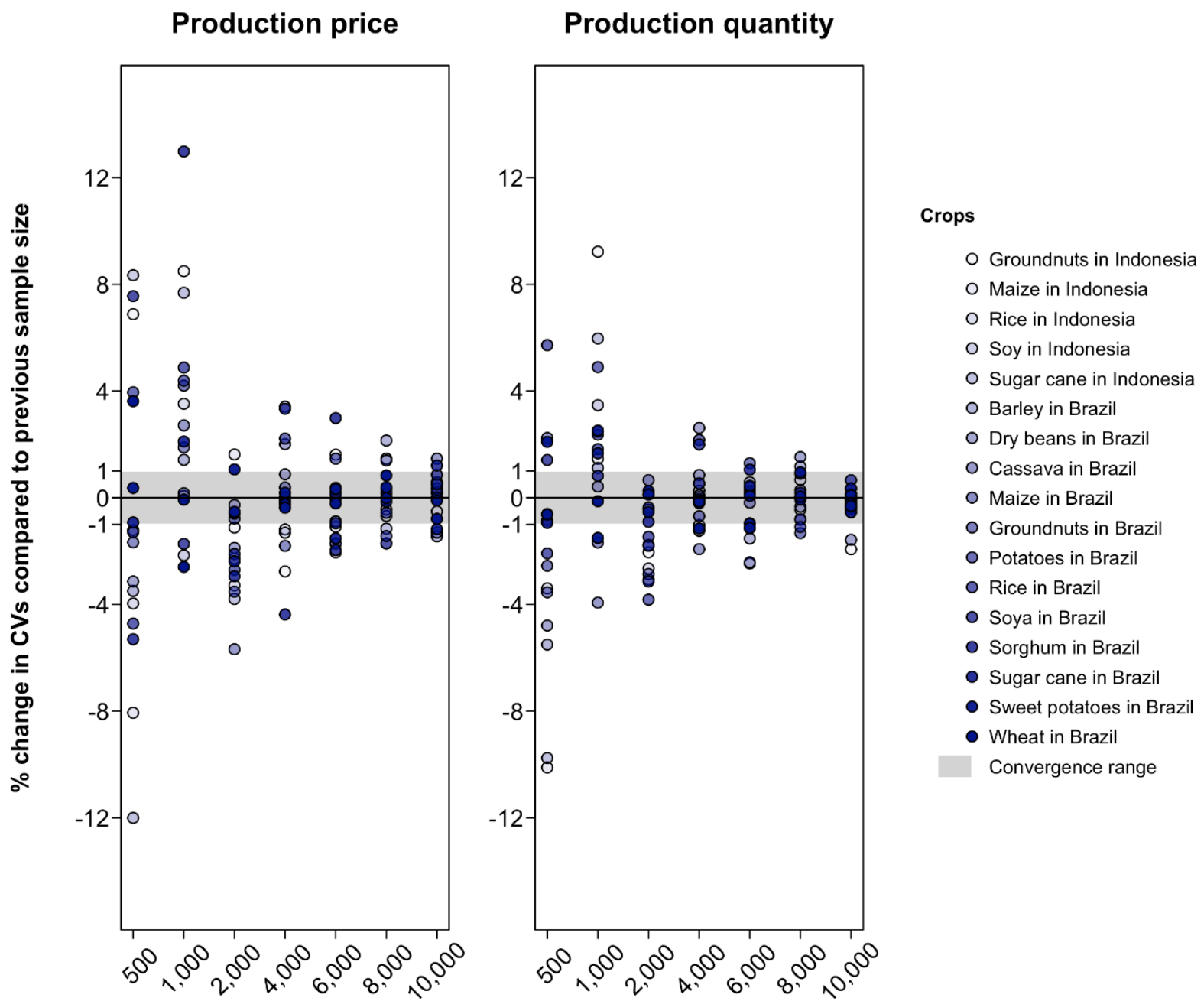

Sample size

Figure A.1. Convergence of the CVs of the production prices and production quantities for GLOBIOM.

The yields of the respective crops are considered uncertain and are randomly drawn from a multivariate normal distribution using the LHS method. The starting sample size is 100 . The sample size is gradually increased until the percentage of changes in the results compared with those obtained from the previous sample size remained within a $\pm 1 \%$ range. 

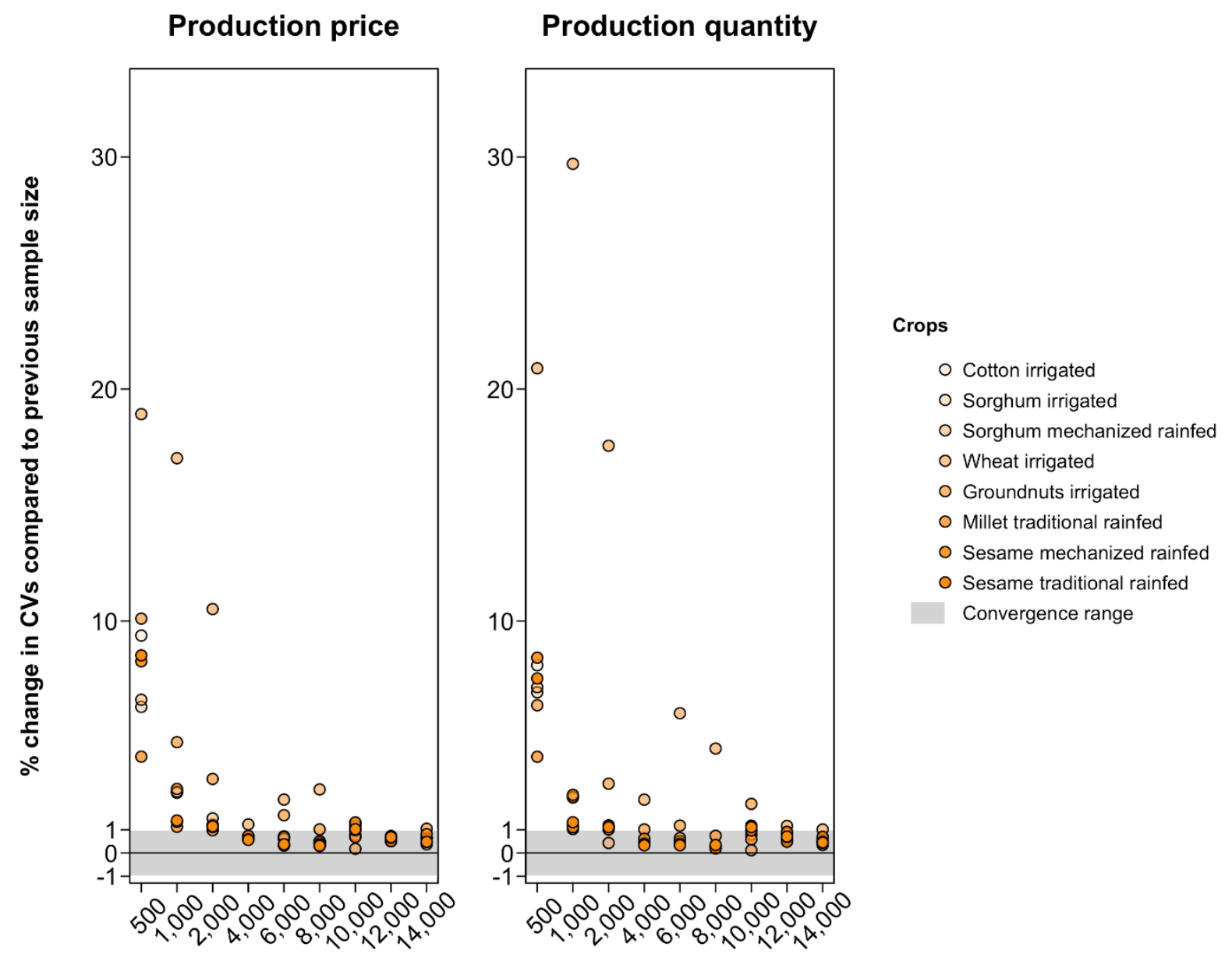

Sample size

\section{Figure A.2. Convergence of the CVs of the average absolute production price and growth rates over the projected period for the dynamic CGE model.}

The total factor productivity parameters of the respective crops are considered uncertain and are randomly drawn from a multivariate normal distribution using the LHS method. The starting sample size is 100 iterations. The sample size is gradually increased until the percentage of changes in the results compared with those obtained from the previous sample size remain within a $\pm 1 \%$ range. 


\section{Appendix B}

a) Production quantity

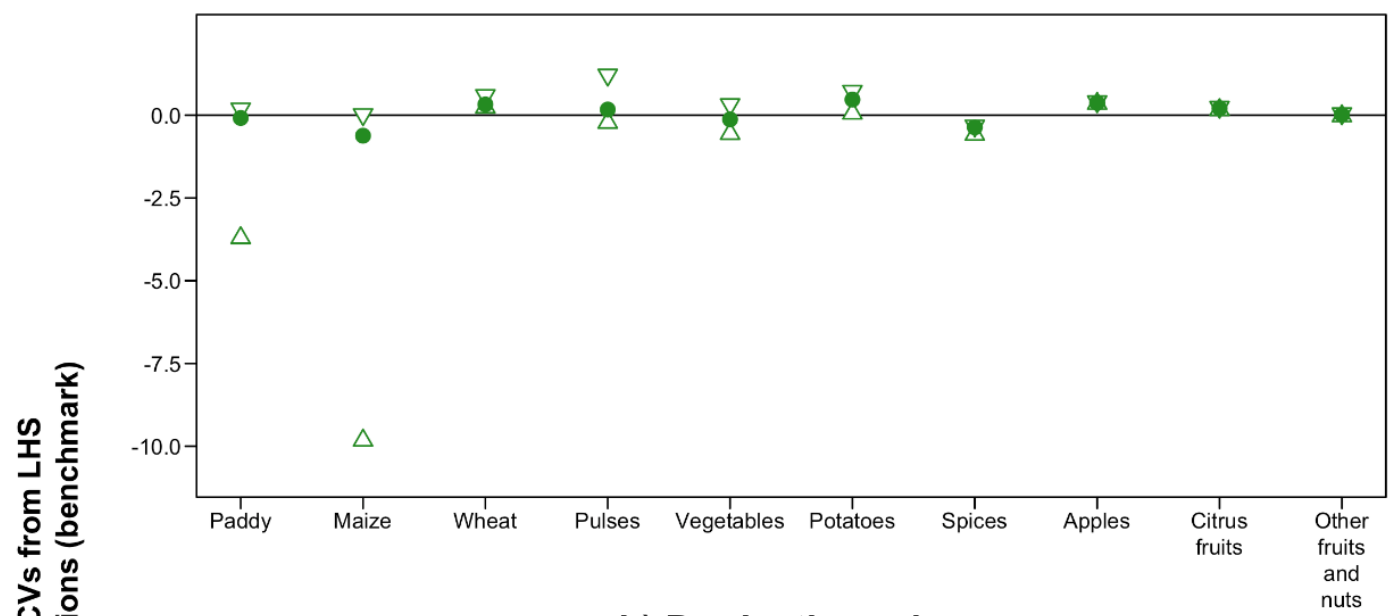

b) Production price

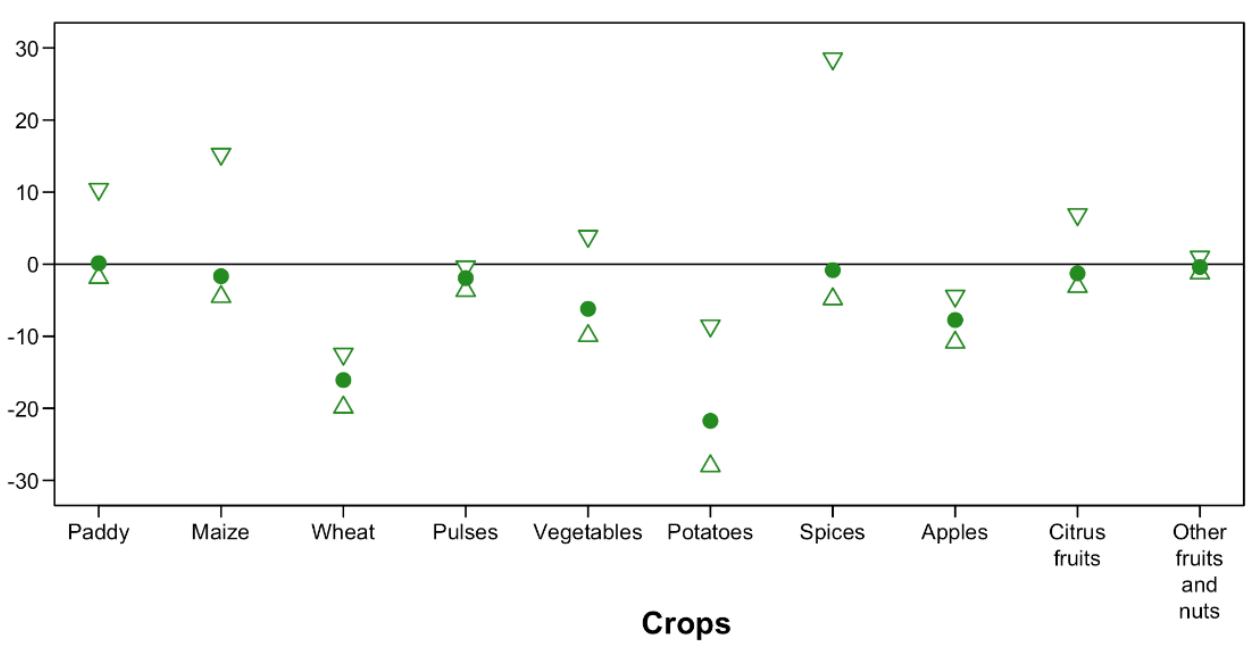

\footnotetext{
- MRGQ (average of all GQ rotations)

$\nabla$ Maximum deviation

$\triangle$ Minimum deviation
}

Figure B.1. Range of deviation of the results produced by single rotations of GQ in the static CGE model.

The deviations are measured in percentage differences of the CVs of the results obtained by each GQ family from the benchmark (LHS with 20,000 iterations). The maximum and minimum deviations for each crop are depicted from 20 randomly generated GQ families. The results obtained by the MRGQ are represented by dots. 
a) Production quantity

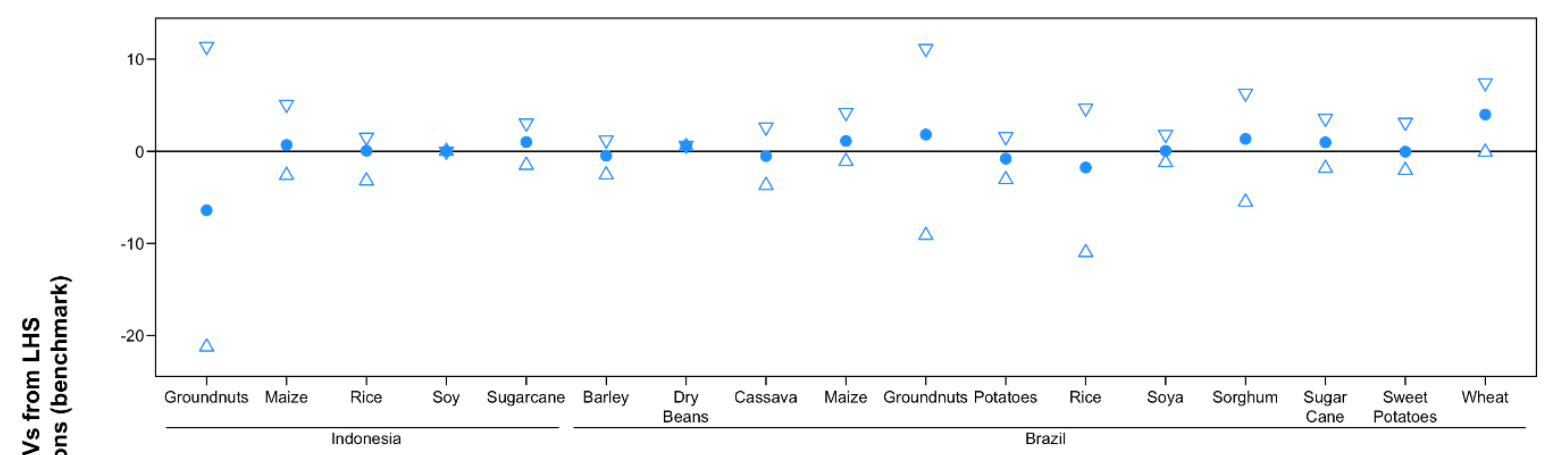

b) Production price

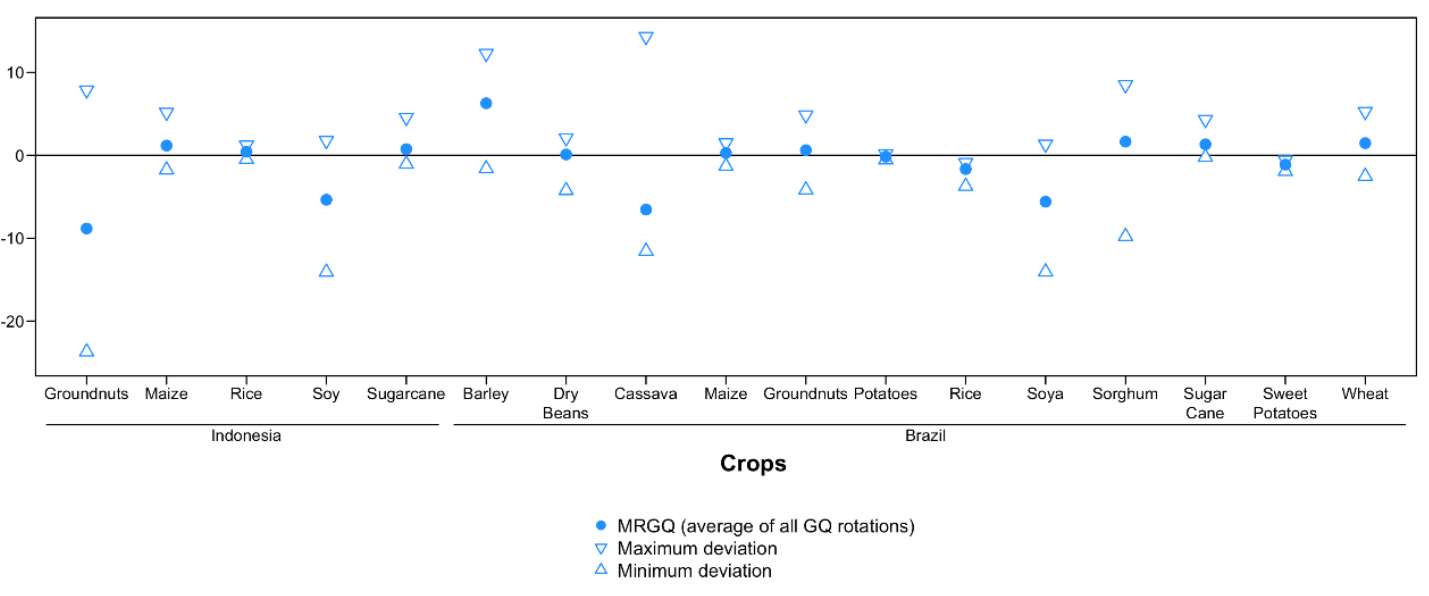

Figure B.2. Range of deviation of the results produced by single rotations of GQ in the GLOBIOM model.

The deviations are measured in percentage differences of the CVs of the results obtained by each GQ family from the benchmark (LHS with 10,000 iterations). The maximum and minimum deviations for each crop are depicted from 10 randomly generated GQ families. The results obtained by the MRGQ are represented by dots. 
a) Production quantity

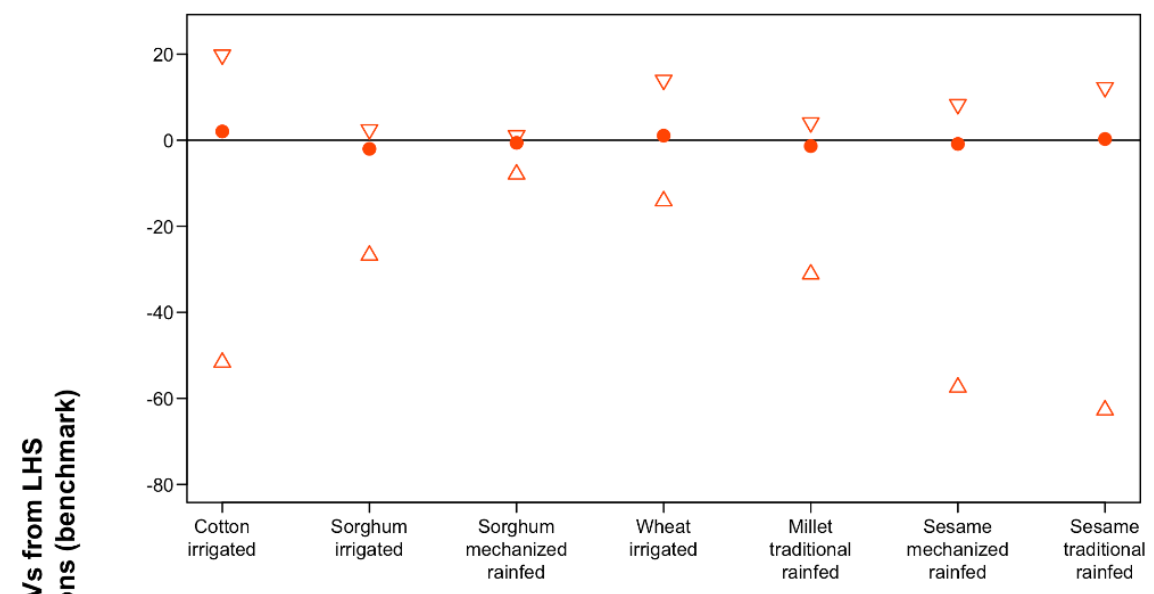

b) Production price

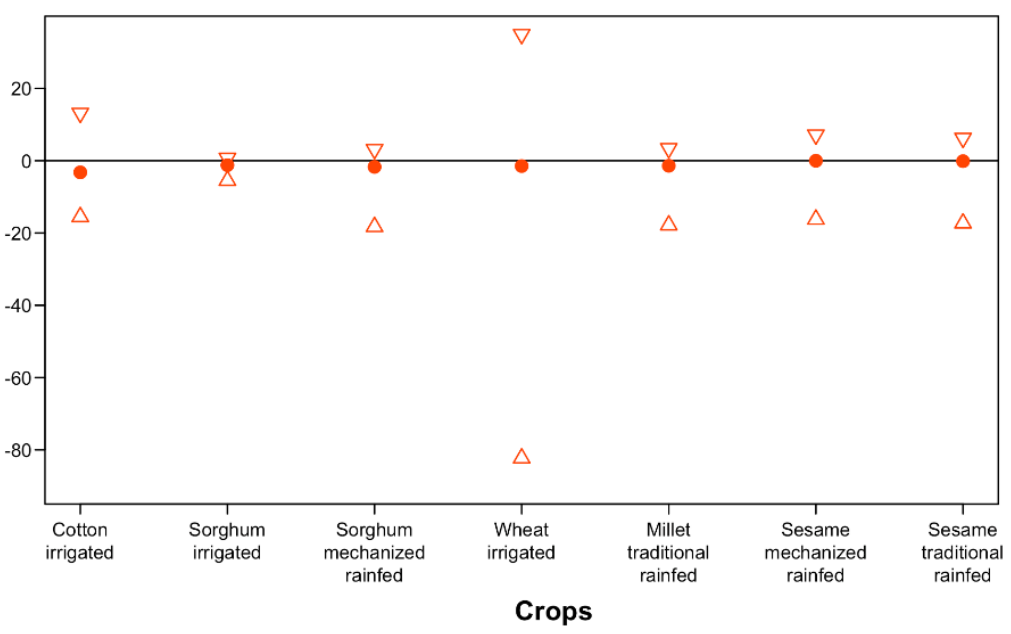

- MRGQ (average of all GQ rotations)
$\nabla$ Maximum deviation

$\triangle$ Minimum deviation

\section{Figure B.3. Range of deviations in the results produced by single rotations of GQ in the} dynamic CGE model.

The deviations are measured in percentage differences of the CVs of the average growth rates of the results obtained by each GQ family from the benchmark during the 2021-2025 period (LHS with 14,000 iterations). The maximum and minimum deviations for each crop are depicted from 20 randomly generated GQ families. The results obtained by the MRGQ are represented by dots. 